\title{
Robust synchronization motion tracking control for a two-slider system restrained by a flexible beam
}

Haibin Dou ( $\nabla$ haibindou1983@163.com )

Binzhou University https://orcid.org/0000-0002-0266-7007

\section{Research Article}

Keywords: sync motion control, disturbance estimation, interval observer, output restriction boundary control, backstepping technology

Posted Date: February 28th, 2022

DOl: https://doi.org/10.21203/rs.3.rs-1397524/v1

License: (c) (i) This work is licensed under a Creative Commons Attribution 4.0 International License. Read Full License 


\title{
Robust synchronization motion tracking control for a two-slider system restrained by a flexible beam \\ Haibin Dou," \\ 1. School of mechatronics engineering, Binzhou University, Binzhou Shandong 256600 haibindou1983@163.com
}

\begin{abstract}
This paper addresses the robust sync tracking control problem for a two-slider system where the motion of two sliders is restrained by a flexible beam. The system models are given at first. In the proposed models the dynamics of actuators are also considered. With employment of the models a novel variable gain sliding mode interval observer is proposed to estimate the external disturbances while reducing the system chattering caused by the conservative fixed gain. Then a tracking controller and an output restriction boundary controller for two sliders are designed. The tracking controller is used to tracking the desired trajectory and the output restriction boundary controller is used to restrict the deformation of the beam. The whole systems with the proposed controller are proved to be asymptotically stable by using traditional backstepping technology. Finally, numerical simulations illustrate that the proposed method are more effective compared with the existing method.
\end{abstract}

Keywords: sync motion control, disturbance estimation, interval observer, output restriction boundary control, backstepping technology

\section{Introduction}

In recent years, synchronization motion control of multiple actuators has attracted more and more interests in industries, such as parallel robots [1]-[3], gantry stage [4]-[6], dual redundant actuation system (DRAS) [7]-[9], coordination control of multi-robots [10]-[12] and so on. In this paper, a special sync system is focused on in which the motion of two actuators is restrained by a mechanism structure. The most typical application of this sync system is the DRAS which is used to promote the reliability and safety of flight control system of the modern airplanes [13]. The sync motion control of this sync system has been introduced in our previous work [11] and [14]. But in [11] the external disturbance is not considered in the design of controller and the proposed controller in [11] and [14] can only deal with the step instruction. So the target of this paper is to realize the robust sync motion tracking control of this sync system.

At present, some researchers have concentrated on exploring the robust sync tracking control of the DRAS. In [15] $\mathrm{Li}$ et al proposed an adaptive robust sync tracking control (ARSTC) approach for a dual redundant hydraulic actuation system with internal leakage fault. In their methods, the coupling models of two control channels are derived with the help of the connection stiffness at first. Then a desired trajectory generator is employed from the dynamics of the control surface. Finally, an ARSTC based on backstepping technology is designed to realize the tracking control. Shi et al [7] proposed a robust fault-tolerant sync tracking control approach for a dissimilar redundant actuation system. The system models are still derived based on the connection stiffness in their methods. Then an active fault-tolerant controller (FTC) based on the performance degradation reference models is given to realize the position track under different hydraulic faults.
Finally, an intelligent matching algorithm is proposed based on the dominant closed-loop poles to obtain the performance degraded fault-tolerant capability. Ijaz et al [16] present an adaptive fault tolerant tracking controller for a DRAS of civil aircraft. In [16] the same force coupling models as in [7] and [15] are used. In order to handle the actuator faults, an adaptive integral sliding mode controller is designed, whose gains can be made adaptive to the fault. Finally, the tracking performance of the proposed controller is validated by comparing the simulations with the existing literatures. Above literatures have a common feature that the connection stiffness is used to describe the coupling force between two control channels. But it is commonly very hard to obtain the connection stiffness from a practical point of view. So in our previous work [11] and [14], a novel coupling force model is derived. To obtain the model, the mechanism structure between two actuators is simplified as a flexible beam. The shear force of the beam is used to represent the coupling force between two actuators. Then two force sensors are used to obtain the shear force instead of the connection stiffness. So the practicability of the force model is greatly improved. This paper will develop a novel robust sync tracking controller based on this model.

To prevent the growth of the controller dimension, the flexible beam is derived by partial differential equations (PDEs), and the models of actuators are described by ordinary differential equations (ODEs). So our models are coupled PDE-ODE models. The researches on boundary disturbances rejection of such models have been introduced in many literatures. Guo et al [17]-[20] proposed a sliding mode control method to solve the problem of input boundary disturbance. But this method needs that the upper bound of boundary disturbance is known. Otherwise, a conservative control 
gain has to be selected. Then the serious system chattering is inevitable. At the same time Guo et al in [19] and [20] extended the active disturbance rejection control (ADRC) to infinite dimensional systems to cope with the boundary input disturbances. But from Feng and Guo in [21], the conventional extended state observer (ESO) used in ADRC has two limitations. One is the derivate of disturbance should be bounded, and the other is a high gain must be needed. So Feng and Guo proposed a new ADRC method to solve these two limitations for a one-dimensional wave equation with boundary disturbance in [21]. In their disturbance estimator, an auxiliary system which separate the disturbance from the original system should be designed to be exponentially stable. Then an infinite dimensional disturbance estimator is designed to be exponentially stable based on the auxiliary system. So the exponential stability should be proved twice in this method by using semigroup theory, which brings some difficulties for practical applications. If the coupled PDE-ODE models are very complex, the proof of stability will more difficult. So this method is not suitable for this paper.

In recent years, the disturbance observer technologies used in ODEs system have been extended to the infinite dimensional system and attracted more and more attention. Jiang et al [22] proposed a disturbance observer for a flexible manipulator in the present of both spatially distributed disturbance and boundary disturbance. But their disturbance observers need an assumption that the spatially distributed disturbance and boundary disturbance are constants which limit their applications. Guo et al [23] designed respectively a basic boundary disturbance observer and an innovative spatially distributed disturbance observer for a flexible marine riser system. Moreover, the well-posedness is also discussed in [23]. Zhao et al [24] proposed a boundary disturbance observer-based controller for a single-link flexible manipulator system with external disturbances. The design process of the disturbance observer is very similar to Guo et al [23]. Zhao and Liu [25] proposed a new finite-time convergence disturbance observer for a flexible Timoshenko manipulator with external disturbances. Compared with [23] and [24], the disturbance estimation error in [25] can converge to zero faster. Compared with above classical disturbance observers which only generate single valued estimates, interval observers (IOs) can produce set-valued estimates under model uncertainties, which can promote the feasibility of disturbance estimation [26]. In [27] Wang et al proposed a novel unknown input IOs for a class of uncertain linear ODE systems to estimate the system states and external disturbance. However, in their estimation method the loose bound of disturbance is used as the coefficient of symbolic function. Though the problem that accurate bounds of disturbances are difficult to be obtained can be solved, conservative control gains have to be selected.

Motivated by [27] and [28], a novel robust sync tracking control approach for a two-slider system restrained by a flexible beam is designed in this paper. This approach consists of two parts. One is the disturbance estimator and the other is sync motion controller. In the proposed disturbance estimator the method in [28] is used to estimate accurately the upper bound of external disturbance. Then the loose bound used in [27] can be replaced by the accurate bound. In this way, the conservativeness of control gains can be improve greatly. In the design of sync controller, one actuator only perform position control and the other suppress the deformation of the beam. The two controllers are both designed based on the ODE backstepping technology and the close-loop system will be prove to be asymptotically stable. The main contributions of this paper are summarized as follows:

(1)A novel variable gain sliding mode interval observer is proposed to estimate the external disturbances, which can avoid the conservativeness of control gains and reduce the system chattering effectively.

(2)An output restriction boundary controller is proposed to confirm that the deformation of the beam is limited within the allowable range. Thus the two actuators can reach the desired position while maintaining sync motion.

The remaining parts of this paper are organized as follows:

The problem formulation and preliminaries are shown in section 2. Section 3 and 4 illustrate the design of disturbance observer and sync motion controller, respectively. Section 5 is the conclusion.

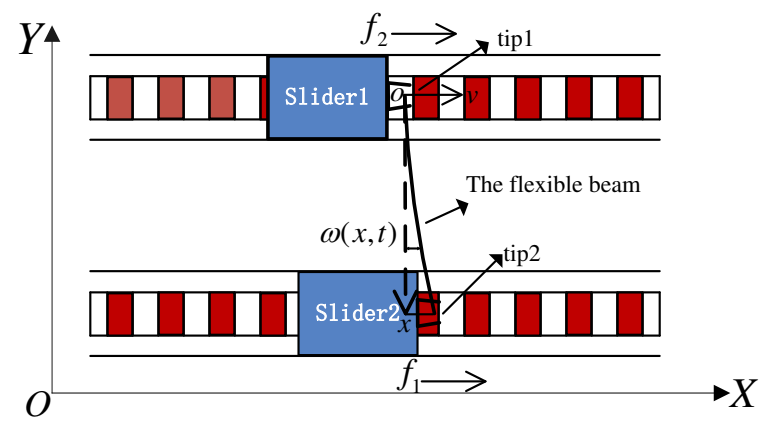

Fig.1 Schematic of the sync motion system

\section{Problem formulation and preliminaries}

\subsection{System dynamics}

The two-slider sync motion system restrained by a flexible beam is displayed in Fig. 1 which consists of two frames. One is frame $O-X Y$ which is used to describe the absolute motion of the beam and sliders and the other is local frame $o-x v$ which is used to describe the deformation of the beam. In the frame $O-X Y$ the two sliders can only move along coordinate $X$. If the positions of two sliders are not synchronous, the deformation of beam in frame $o-x v$ will occur. $f_{1}(t)$ and $f_{2}(t)$ in Fig. 1 are the interaction forces between sliders and beam. From our previous work [14] and [29], the models of two-slider system can be described as 


$$
\left\{\begin{array}{l}
\rho(t)+(t, t))+E I \omega^{\prime \prime \prime \prime}(x, t)=0 \\
m(t)=f_{1}(t)+f_{2}(t) \\
x_{2}(t)=x_{1}(t)+\omega(l, t) \\
M_{1}(t)=a_{1} x(t)+a_{2} \varpi_{1}(t) \\
\quad-f_{1}(t)-E I \omega^{\prime \prime \prime}(0, t)+d_{11}(t) \\
M_{2}(t)=b_{1} d(t)+b_{2} \varpi_{2}(t) \\
\quad-f_{2}(t)+E I \omega^{\prime \prime \prime}(l, t)+d_{21}(t) \\
\omega(0, t)=\omega^{\prime}(0, t)=\omega^{\prime \prime}(l, t)=0
\end{array}\right.
$$

where $\rho, E I$ and $l$ are the uniform linear mass density, uniform flexural rigidity and length respectively, $x_{1}(t)$ and $x_{2}(t)$ are the positions of two sliders respectively, $\omega(x, t)$ is the deformation of the beam, $x \in[0, l]$ and $t$ are independent space and time variables, $m, M_{1}$ and $M_{2}$ describe the mass of the beam, slider 1 and slider 2 respectively, $a_{2} \varpi_{1}(t)$ and $b_{2} \varpi_{2}(t)$ are the input forces, $a_{1}$ and $b_{1}$ are damping coefficients, $d_{11}(t)$ and $d_{21}(t)$ are external disturbances, $E I \omega^{\prime \prime \prime}(0, t)$ and $E I \omega^{\prime \prime \prime}(l, t)$ represent the shear force at end 0 and end $l$ respectively. In [14] and [29] the dynamics of sliders are not considered. Here consider the following dynamics of sliders as

$$
\left\{\begin{array}{l}
\phi_{1}(t)=a_{3} \&(t)+a_{4} \theta_{1}(t)+K_{1} u_{1}(t)+d_{12}(t) \\
\oiint_{2}(t)=b_{3} \&(t)+b_{4} \theta_{2}(t)+K_{2} u_{2}(t)+d_{22}(t)
\end{array}\right.
$$

where $a_{3}, a_{4}, b_{3}, b_{4}, K_{1}$ and $K_{2}$ are constants, $u_{1}(t)$ and $u_{2}(t)$ are input signals, $d_{12}(t)$ and $d_{22}(t)$ are external disturbances.

Combining Eq.(1) and (2), the whole system models are as follows

$$
\begin{gathered}
\rho(t)+(x, t))+E I \omega^{\prime \prime \prime \prime}(x, t)=0 \\
\omega(0, t)=\omega^{\prime}(0, t)=\omega^{\prime \prime}(l, t)=0 \\
\bar{M}_{1}(t)=a_{1} \&(t)+a_{2} \theta_{1}(t) \\
+f_{2}(t)-E I \omega^{\prime \prime \prime}(0, t)+d_{11}(t) \\
\&(t)=a_{3} \&(t)+a_{4} \theta_{1}(t)+K_{1} u_{1}(t)+d_{12}(t) \\
M_{2}(t)=b_{1} \&(t)+b_{2} \theta_{2}(t) \\
-f_{2}(t)+E I \omega^{\prime \prime \prime}(l, t)+d_{21}(t) \\
\&(t)=b_{3} x_{2}(t)+b_{4} \theta_{2}(t)+K_{2} u_{2}(t)+d_{22}(t) \\
x_{2}(t)=x_{1}(t)+\omega(l, t)
\end{gathered}
$$

where $\bar{M}_{1}=m+M_{1}$, the shear force can be obtained as follows from [11]

$$
\left\{\begin{array}{l}
E I \omega^{\prime \prime \prime}(0, t)=\frac{f_{1}(t)-f_{2}(t)}{2} \\
E I \omega^{\prime \prime \prime}(l, t)=\frac{f_{2}(t)-f_{1}(t)}{2}
\end{array}\right.
$$

In the subsequent sections the following assumptions are needed.

Assumption 1: The external disturbances and their time derivative satisfy that $\left|d_{11}(t)\right|<\bar{d}_{11}, \quad\left|d_{12}(t)\right|<\bar{d}_{12}$, $\left|d_{21}(t)\right|<\bar{d}_{21}, \quad\left|d_{22}(t)\right|<\bar{d}_{22}, \quad\left|\&_{11}^{\&}(t)\right|<L_{11}, \quad\left|d_{12}^{\&}(t)\right|<L_{12}$, $\left|\mathscr{d}_{21}^{\&}(t)\right|<L_{21}$ and $\left|\&_{22}^{\&}(t)\right|<L_{22}$, where $\bar{d}_{11}, \bar{d}_{12}, \bar{d}_{21}$, $\bar{d}_{22}, \quad L_{11}, L_{12}, \quad L_{21}$ and $L_{22}$ are all unknown constants.

Assumption 2: The shear force $E I \omega^{\prime \prime \prime}(0, t)$ and $E I \omega^{\prime \prime \prime}(l, t)$ are both bounded.

Remark 1: The Assumption 2 is reasonable. The output forces of two sliders are usually bounded from the practical point of view. Then by using Eq.(4) the shear force $E I \omega^{\prime \prime \prime}(0, t)$ and $E I \omega^{\prime \prime \prime}(l, t)$ are both bounded.

\subsection{Preliminaries}

In subsequent sections the following lemmas are needed.

Lemma 1: [27] If a vector $x$ satisfies that $\underline{x} \preccurlyeq x \leqslant \bar{x}$, then the following inequality holds for matrix $A$

$$
A^{+} \underline{x}-A^{-} \bar{x} \preccurlyeq A x \preccurlyeq A^{+} \bar{x}-A^{-} \underline{x}
$$

Remark 2: For matrixes $A=\left(a_{i j}\right)_{m \times n}$ and $B=\left(b_{i j}\right)_{m \times n}$, the symbols " $\preccurlyeq "$ or " $\succcurlyeq "$ represent the element-wise relation, for example, $A \geqslant B$ means $a_{i j} \geq b_{i j}, i=1, \ldots, m$, $j=1, \ldots, n$. The matrix $A^{+}=\left(a_{i j}^{+}\right)_{m \times n}$ means $a_{i j}^{+}=$ $\max \left\{0, a_{i j}\right\}$. Define $A^{-}=A^{+}-A$.

Lemma 2: [30] if matrix pair $(N, M)$ is detectable, a gain $L$ and a nonsingular transformation matrix $S$ can be found to confirm that $M-L N$ is Hurwitz and $S(M-L N) S^{-1}$ is Metzler.

Lemma 3: [31] A linear system is described as $\&(t)=A x(t)+b(t)$ and its initial value satisfies that $x(0)=x_{0}$. If $A$ is Metzler , $b(t) \geqslant 0$ and $x_{0} \geqslant 0$, then $x(t) \geqslant 0$ for all $t \geq 0$.

Lemma 4: [28, 32] A dynamic system is described as

$$
\&(t)=a(t)+u(t)
$$

where $a(t)$ is the disturbance and satisfies that $|a(t)| \leq \varsigma_{1}$ and $|\&(t)| \leq \varsigma_{2}, \quad \varsigma_{1}$ and $\varsigma_{2}$ are both unknown constants. If the control input $u(t)$ is designed as

$$
u(t)=-(k(t)+\eta) \operatorname{sign}(\Omega(t))
$$

where $\eta$ is a positive constant and $k(t)$ is updated by the adaptive law:

$$
\begin{aligned}
& \&(t)=-\left(r(t)+r_{0}\right) \operatorname{sign}(\delta(t)) \\
& \&(t)= \begin{cases}\gamma|\delta(t)|, & \text { if } \delta(t)>\delta_{0} \\
0, & \text { otherwise }\end{cases}
\end{aligned}
$$

where $\quad \delta(t)=k(t)-\left|u_{e q}(t)\right| / \alpha-b \quad, \quad \iota_{e q}(t)=(u(t)-$ $\left.u_{e q}(t)\right) / \tau$, the parameters $0<\alpha<1, \delta_{0}, \gamma, b$ and $q>\sup \left(1,\left|\iota_{e q}(t)\right| / \varsigma_{2}\right)$ are selected to satisfy that

$$
\frac{1}{4} b^{2}>\delta_{0}^{2}+\frac{1}{\gamma}\left(\frac{q \varsigma_{2}}{\alpha}\right)^{2}
$$

Then $k(t)>|a(t)|$ in finite-time can be obtained. 
Moreover, $k(t)$ and $r(t)$ are both bounded.

Lemma 5: [35] Introduce the first-order Levant differentiator as follows:

$$
\begin{aligned}
& \phi_{1}(t)=\vartheta_{1}(t) \\
& \vartheta_{1}(t)=-\gamma_{1}\left|\varphi_{1}(t)-\beta_{r}(t)\right|^{1 / 2} \operatorname{sign}\left(\varphi_{1}(t)-\beta_{r}(t)\right)+\varphi_{2}(t) \\
& \psi_{2}(t)=-\gamma_{2} \operatorname{sign}\left(\varphi_{2}(t)-\vartheta_{1}(t)\right)
\end{aligned}
$$

where $\beta_{r}(t)$ is the input signal. Properly choosing the parameters $\gamma_{1}$ and $\gamma_{2}$ we have that the following equalities are true in absence of input noises

$$
\varphi_{1}(t)=\beta_{r}(t), \vartheta_{1}(t)=\beta_{r}^{\&}(t)
$$

Lemma 6: [22] Let $\Theta(s, t) \in R$, where $t$ and $s$ satisfy that $t \in[0, \infty)$ and $s \in[0, l]$. If $\Theta(0, t)=0$ is also satisfied, then we have

$$
\Theta^{2}(s, t) \leq l \int_{0}^{l}\left[\Theta^{\prime}(s, t)\right]^{2} d s
$$

Lemma 7: [29] If $\Theta(s, t)$ is continuously differentiable on $s \in[0, S]$ and $\Theta(0, t)=\Theta^{\prime}(0, t)=0$, then we have

$$
\int_{0}^{S} \Theta^{2}(s, t) d s \leq S^{2} \int_{0}^{S}\left[\Theta^{\prime}(s, t)\right]^{2} d s \leq S^{4} \int_{0}^{S}\left[\Theta^{\prime \prime}(s, t)\right]^{2} d s
$$

\section{Sliding mode interval observer design}

In this section, a variable gain sliding mode interval observer will be proposed to estimate the external disturbances in Eq.(3). The observers of the first control channel in Eq.(3) is the same as those of the second control channel. Therefore, only the observers of slider 1 is given in this section. At first the dynamics of slider 1 in Eq.(3) are converted into descriptor form as follows

$$
\&(t)=A \mathrm{x}_{1}(t)+B u_{1}+W_{1}(t)+T_{1}(t)
$$

where $\mathrm{x}_{1}(t)=\left[x_{1}, \&, \theta_{1}\right]^{T}, \quad B=\left[0 ; 0 ; K_{1}\right], \quad W_{1}(t)=$ $\left[0 ; \frac{f_{2}-E I \omega^{\prime \prime \prime}(0, t)}{\bar{M}_{1}} ; 0\right], T_{1}=\left[0 ; \frac{1}{\bar{M}_{1}} d_{11}(t) ; d_{12}(t)\right]$, $A=\left[\begin{array}{ccc}0 & 1 & 0 \\ 0 & a_{1} / \bar{M}_{1} & a_{2} / \bar{M}_{1} \\ 0 & a_{3} & a_{4}\end{array}\right]$.

Introducing a nonsingular state transformation $\left[\mathrm{x}_{T_{1}} ; \mathrm{x}_{T_{2}}\right]=T \mathrm{x}_{1}$ for Eq.(5) yields

$$
\begin{aligned}
\&= & A_{1} \mathrm{x}_{T_{1}}+B_{1} u_{1}+W_{T}+d_{T} \\
& \&=A_{3} \mathrm{x}_{T_{1}}
\end{aligned}
$$

where $\mathrm{x}_{T_{1}}=\left[\&(t) ; \theta_{1}(t)\right], \quad \mathrm{x}_{T_{2}}=\left[x_{1}(t)\right], \quad B_{1}=\left[0 ; K_{1}\right]$, $W_{T}=\left[\frac{f_{2}-E I \omega^{\prime \prime \prime}(0, t)}{\bar{M}_{1}} ; 0\right], \quad d_{T}=\left[\frac{d_{11}(t)}{\bar{M}_{1}} ; d_{12}(t)\right]$, $A_{1}=\left[\begin{array}{cc}a_{1} / \bar{M}_{1} & a_{2} / \bar{M}_{1} \\ a_{3} & a_{4}\end{array}\right], \quad A_{3}=\left[\begin{array}{ll}1 & 0\end{array}\right], \quad T=\left[\begin{array}{lll}0 & 1 & 0 \\ 0 & 0 & 1 \\ 1 & 0 & 0\end{array}\right]$.

By using some direct calculations we have that the pair $\left(A_{3}, A_{1}\right)$ is detectable. From Lemma 2 there exists $V_{1}$ and $S_{1}$ such that $V_{1}\left(A_{1}+S_{1} A_{3}\right) V_{1}^{-1}$ is Hurwitz and
Metzler. Define a new variable as $\eta_{1}(t)=V_{1}\left(\mathrm{x}_{T_{1}}+S_{1} \mathrm{x}_{T_{2}}\right)=\left\{\eta_{1,1} ; \eta_{1,2}\right\}$, then from Eq.(6) its time derivative is

$$
1 \&(t)=A_{L} \eta_{1}(t)-A_{N} \mathrm{x}_{T_{2}}+V_{1} B_{1} u_{1}+V_{1} W_{T}+V_{1} d_{T}
$$

where $A_{L}=V_{1}\left(A_{1}+S_{1} A_{3}\right) V_{1}^{-1}$ and $A_{N}=V_{1}\left(A_{1}+S_{1} A_{3}\right) S_{1}$. From Lemma 1 and Assumption 1, we have

$$
V_{1}^{+} \underline{d_{T}}-V_{1}^{-} \bar{d}_{T} \preccurlyeq V_{1} d_{T} \preccurlyeq V_{1}^{+} \bar{d}_{T}-V_{1}^{-} \underline{d_{T}}
$$

where $\underline{d}_{T} \preccurlyeq d_{T} \preccurlyeq \bar{d}_{T}$.

Then from Eq.(7) the IOs can be designed as

$\mathcal{H}_{1}^{f}(t)=A_{L} \bar{\eta}_{1}(t)-A_{N} \mathrm{x}_{T_{2}}+V_{1} B_{1} u_{1}+V_{1} W_{T}+V_{1}^{+} \bar{d}_{T}-V_{1}^{-} \underline{d_{T}}$

$\underline{1 \&}(t)=A_{L} \underline{\eta_{1}}(t)-A_{N} \mathrm{x}_{T_{2}}+V_{1} B_{1} u_{1}+V_{1} W_{T}+V_{1}^{+} \underline{d_{T}}-V_{1}^{-} \bar{d}_{T}$

where $\bar{\eta}_{1}(t)=\left\{\bar{\eta}_{1,1}(t) ; \bar{\eta}_{1,2}(t)\right\}$ and $\underline{\eta_{1}}(t)=\left\{\underline{\eta_{1,1}}(t) ; \underline{\eta_{1,2}}(t)\right\}$. Using Eq.(8) and (9) the following lemma can be obtained.

Lemma 8: $\eta_{1}(t)$ is bounded by $\underline{\eta_{1}}(t) \preccurlyeq \eta_{1}(t) \preccurlyeq \bar{\eta}_{1}(t)$.

Proof. Define $\bar{e}_{\eta_{1}}=\bar{\eta}_{1}-\eta_{1}$ and $\underline{e_{\eta_{1}}}=\eta_{1}-\underline{\eta_{1}}$. Using Eq.(7)-(9) we have

$$
\begin{aligned}
& \stackrel{\mathrm{d}}{\mathrm{\eta}_{1}}=A_{L} \bar{e}_{\eta_{1}}+V_{1}^{+} \bar{d}_{T}-V_{1}^{-} \underline{d_{T}}-V_{1} d_{T} \\
& \underline{\boldsymbol{q}_{1}}=A_{L} \underline{e_{\eta_{1}}}+V_{1} d_{T}-V_{1}^{+} \underline{d_{T}}+V_{1}^{-} \bar{d}_{T}
\end{aligned}
$$

It is clear that $A_{L}$ is Metzler, $V_{1}^{+} \bar{d}_{T}-V_{1}^{-} \underline{d_{T}}-V_{1} d_{T} \geqslant 0$, $V_{1} d_{T}-V_{1}^{+} \underline{d_{T}}+V_{1}^{-} \bar{d}_{T} \geqslant 0, \quad \bar{e}_{\eta_{1}}(0), \quad \underline{e_{\eta_{1}}}(0) \geqslant 0$. Then from Lemma $3 \quad \bar{e}_{\eta_{1}}(t), \underline{e_{\eta_{1}}}(t) \geqslant 0$ can be obtained.

By using Eq.(8) and (9) define the estimation of $\eta_{1}(t)$ as

$$
\hat{\eta}_{1}(t)=\Pi_{1} \bar{\eta}_{1}+\left(I_{2}-\Pi_{1}\right) \underline{\eta_{1}}
$$

where $I_{2} \in R^{2 \times 2}$ is an identity matrix, $\Pi_{1}=\operatorname{diag}\left(\lambda_{i}\right)$, $i=1,2$. The adaptive law for $\lambda_{i}(t)$ is designed as follows

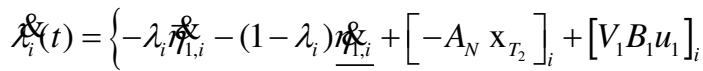

$$
\begin{aligned}
& \left.+\left[V_{1} W_{T}\right]_{i}+\mu_{i} \operatorname{sign}\left(\eta q_{1, i}\right)+\left[A_{L} \hat{\eta}_{1}\right]_{i}\right\} /\left(\bar{\eta}_{1, i}-\underline{\eta_{1, i}}\right)
\end{aligned}
$$

where $\eta q_{1, i}=\eta_{1, i}-\hat{\eta}_{1, i},[\bullet]_{i}$ means the $i$ th element of vector $\bullet, i=1,2$. In the next, the variable gain $\mu_{i}$ will be designed to avoid the conservativeness.

Introduce two new variables $\Omega_{1}(t)$ and $\Omega_{2}(t)$ as

$$
\begin{aligned}
& \Omega_{1}(t)=\&(t)-\sigma_{1}(t) \\
& \Omega_{2}(t)=\theta_{1}(t)-\sigma_{2}(t)
\end{aligned}
$$

where

$$
\begin{gathered}
\bar{M}_{1} \&(t)=-E I \omega^{\prime \prime \prime}(0, t)+f_{2}+a_{1} \cdot \&(t)+a_{2} \theta_{1}(t)-v_{1}(t), \\
\alpha_{2}^{\&}(t)=a_{3} \&(t)+a_{4} \theta_{1}(t)+K_{1} u_{1}-v_{2}(t),
\end{gathered}
$$

where $v_{1}(t)=-\left(k_{1}(t)+k_{10}\right) \operatorname{sign}\left(\Omega_{1}\right), \quad k_{10}$ and $k_{20}$ are small positive constants, $v_{2}(t)=-\left(k_{2}(t)+k_{20}\right) \operatorname{sign}\left(\Omega_{2}\right)$, $k_{1}(t)$ and $k_{2}(t)$ will be designed in the following. 
The time derivative of Eq.(11) and (12) are as follows

$$
\begin{aligned}
& \bar{M}_{1} \bigotimes_{1}(t)=\bar{M}_{1} \&(t)-\bar{M}_{1} \sigma_{1}(t) \\
& =a_{1} \&(t)+a_{2} \theta_{1}(t)+f_{2}(t)-E I \omega^{\prime \prime \prime}(0, t)+d_{11}(t) \\
& -\left[-E I \omega^{\prime \prime \prime}(0, t)+f_{2}+a_{1} \cdot \&(t)+a_{2} \theta_{1}(t)-v_{1}(t)\right] \\
& =d_{11}(t)+v_{1}(t) \\
& \oiint_{2}(t)=\&_{1}(t)-\mathbb{\alpha}_{2}(t) \\
& =a_{3} \&(t)+a_{4} \theta_{1}(t)+K_{1} u_{1}+d_{12}(t) \\
& -\left[a_{3} \cdot \&(t)+a_{4} \theta_{1}(t)+K_{1} u_{1}-v_{2}(t)\right] \\
& =d_{12}(t)+v_{2}(t)
\end{aligned}
$$

Then the two-layer adaptive laws for $k_{1}(t)$ and $k_{2}(t)$ are designed as follows

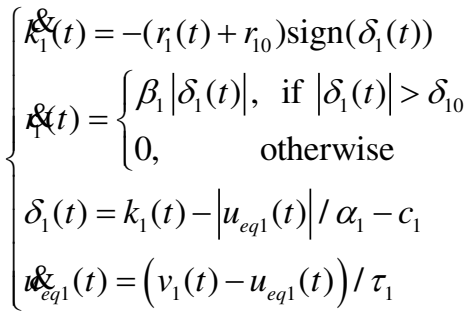

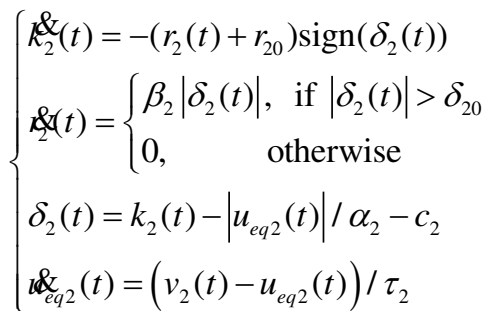

where the parameters $r_{10}, r_{20}, \beta_{1}, \beta_{2}, \delta_{10}, \delta_{20}$, $\alpha_{1}, \alpha_{2}, c_{1}, c_{2}, \tau_{1}$ and $\tau_{2}$ are all positive constants, and they satisfy that

$$
\left\{\begin{array}{l}
0<\alpha_{1}, \alpha_{2}<1 \\
\frac{c_{1}^{2}}{4}>\delta_{10}^{2}+\frac{1}{\beta_{1}}\left(\frac{q_{1} L_{11}}{\alpha_{1}}\right)^{2} \\
\frac{c_{2}^{2}}{4}>\delta_{20}^{2}+\frac{1}{\beta_{2}}\left(\frac{q_{2} L_{12}}{\alpha_{2}}\right)^{2} \\
q_{1}>\sup \left(1, \frac{\left|\&_{\text {eq } 1}\right|}{L_{11}}\right), q_{2}>\sup \left(1, \frac{\left|\&_{\text {eq }}\right|}{L_{12}}\right)
\end{array}\right.
$$

Then from Lemma $4\left|d_{11}(t)\right|<k_{1}(t)$ and $\left|d_{12}(t)\right|<k_{2}(t)$ can be obtained. Now the gains $\mu_{i}$ in Eq.(10) can be designed as

$$
\mu_{i}=\max \left\{\left|V_{1}^{+} \underline{d_{T}}-V_{1}^{-} \bar{d}_{T}\right|_{i},\left|V_{1}^{+} \bar{d}_{T}-V_{1}^{-} \underline{d_{T}}\right|_{i}\right\}
$$

where $d_{T}=\left[\frac{-k_{1}(t)}{\bar{M}_{1}} ;-k_{2}(t)\right]$ and $\bar{d}_{T}=\left[\frac{k_{1}(t)}{\bar{M}_{1}} ; k_{2}(t)\right]$. From Eq.(10) the time derivative of $\eta_{1, i}$ can be obtained as

$$
\begin{aligned}
& \overbrace{1, i}^{\&}=1 \%, r i \\
& =\left[A_{L} \eta_{1}(t)\right]_{i}-\left[A_{N} \mathrm{x}_{T_{2}}\right]_{i}+\left[V_{1} B_{1} u_{1}\right]_{i}+\left[V_{1} W_{T}\right]_{i}+\left[V_{1} d_{T}\right]_{i}
\end{aligned}
$$

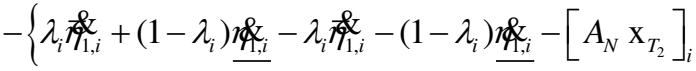

$$
\begin{aligned}
& \left.+\left[V_{1} B_{1} u_{1}\right]_{i}++\left[V_{1} W_{T}\right]_{i}+\mu_{i} \operatorname{sign}\left(\eta q_{1, i}\right)+\left[A_{L} \hat{\eta}_{1}\right]_{i}\right\} \\
& =\left[A_{L} r q(q)\right]_{i}+\left[V_{1} d_{T}\right]_{i}-\mu_{i} \operatorname{sign}\left(\eta q_{1, i}\right)
\end{aligned}
$$

By using Eq.(17) Theorem 1 can be obtained as follows Theorem 1: The proposed observer $\hat{\eta}_{1}(t)$ can confirm that the estimation error $\eta_{1}(t)$ converges to zero as $t \rightarrow \infty$. Then the external disturbances can be estimated by

$$
\hat{d}_{T}=V_{1}^{-1}(\mu \cdot \operatorname{sign}(\eta q))_{e q}
$$

where $(\bullet)_{e q}$ is the equivalent output injection signal, $\mu=\operatorname{diag}\left[\mu_{i}\right], i=1,2$.

Proof. Construct a Lyapunov function $V_{\eta_{1}}(t)$ as

$$
V_{\eta_{1}}(t)=\frac{1}{2} r \eta_{1} \text { or } \eta_{1}
$$

From Eq.(17) its time derivative is

$$
\begin{aligned}
& v_{\eta_{1}}=1 q_{1}^{7} \% \\
& =\eta \% \%\left(A_{L} r q d(t)+V_{1} d_{T}-\mu \operatorname{sign}\left(7 q_{1}\right)\right)
\end{aligned}
$$

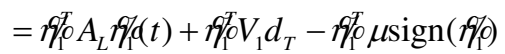

$$
\begin{aligned}
& \leq \eta q_{1}^{T} A_{L} \eta q_{1}(t)+\left|r q_{1,1}\right|\left(\left|V_{1} d_{T}\right|_{1}-\mu_{1}\right)+\left|r q_{1,2}\right|\left(\left|V_{1} d_{T}\right|_{2}-\mu_{2}\right) \\
& \leq \eta q_{1}^{T} A_{L} \eta q_{1}(t) \leq 0
\end{aligned}
$$

Therefore, the estimation error $\eta_{1} /(t)$ can converge to zero as $t \rightarrow \infty$. When $19 /(t)$ becomes very small, we can approximately have

$$
r_{1}(t)=\eta q_{1}(t) \approx 0
$$

Then by using the equivalent output error injection [33] the external disturbance can be estimate approximately by

$$
V_{1} d_{T} \approx[\mu \cdot \operatorname{sign}(\eta / \%)]_{e q}
$$

where $[\bullet]_{e q}$ can be obtained by passing $\bullet$ into a low-pass filter, i.e., $\tau\left(\varphi_{e q}+(\bullet)_{e q}=(\bullet), \tau\right.$ is the time constant of the filter. Eq.(18) can be converted into

$$
\hat{d}_{T}=V_{1}^{-1}\left[\mu \cdot \operatorname{sign}\left(\eta \eta_{1}\right)\right]_{e q}
$$

The proof is completed.

\section{Sync motion tracking control design}

In this section, a traditional backstepping technology based on Eq.(3) will be used to design the sync motion controller to confirm two sliders can track the desired trajectory while restraining the deformation of the beam. The tracking controller for slider 1 is given at first.

\subsection{Robust tracking controller for slider 1}

For Eq.(3c) define tracking error $z_{1}$ as $z_{1}=x_{1}-x_{d}$, where $x_{d}$ is the desired trajectory.

Step1: Construct the Lyapunov function $V_{1}$ as $V_{1}=z_{1}^{2} / 2$. 
Its time derivative is $V_{1}^{\&}=z_{1} \&$. Introduce the virtual control input $\alpha_{1}(t)$. Then define the tracking error $z_{2}(t)$ as

$$
z_{2}=\&-\alpha_{1}
$$

where $\alpha_{1}=-c_{1} z_{1}+\&, c_{1}$ is a positive control gain.

By using Eq.(19) $V_{1}^{\&}$ becomes

$$
\nu_{1}^{\&}=z_{1}\left(z_{2}+\alpha_{1}-\underset{d}{\&}\right)=z_{1} z_{2}-c_{1} z_{1}^{2}
$$

Step 2: Construct Lyapunov function $V_{2}$ as

$$
V_{2}=V_{1}+\bar{M}_{1} z_{2}^{2} / 2
$$

Its time derivative is

$$
\begin{aligned}
V_{2}^{\&}= & z_{1} z_{2}-c_{1} z_{1}^{2}+\bar{M}_{1} z_{2} \& \\
= & z_{1} z_{2}-c_{1} z_{1}^{2}+z_{2}\left[a_{1} \&+a_{2} \theta_{1}+f_{2}\right. \\
& \left.-E I \omega^{\prime \prime \prime}(0, t)+d_{11}(t)-\bar{M}_{1} \& \mathrm{~F}\right]
\end{aligned}
$$

Introduce the virtual control input $\alpha_{2}(t)$ and define the tracking error $z_{3}$ as $z_{3}=\theta_{1}(t)-\alpha_{2}(t)$, where $\alpha_{2}$ is designed as

$$
\begin{aligned}
\alpha_{2}(t)=\left(1 / a_{2}\right)[ & -a_{1} \&-f_{2}+E I \omega^{\prime \prime \prime}(0, t) \\
& \left.-\hat{d}_{11}-z_{1}-c_{2} z_{2}+\bar{M}_{1} \&\right]
\end{aligned}
$$

Substituting Eq.(22) into Eq.(21) yields

$$
\begin{aligned}
v_{2}^{\&} & =-c_{1} z_{1}^{2}-c_{2} z_{2}^{2}+a_{2} z_{2} z_{3}+z_{2} d_{11}^{d o} \\
& \leq-c_{1} z_{1}^{2}-\left(c_{2}-\frac{1}{2}\right) z_{2}^{2}+a_{2} z_{2} z_{3}+\frac{1}{2} d_{11}^{8}
\end{aligned}
$$

where $c_{2}$ is a positive control gain, $d_{11}^{o}=d_{11}-\hat{d}_{11}$.

Step 3: Construct the Lyapunov function as

$$
V_{3}=V_{1}+V_{2}+z_{3}^{2} / 2
$$

Its time derivative is

$$
\begin{aligned}
& V_{3}^{\&}=V_{1}^{\&}+V_{2}^{\&}+z_{3} \& \\
& =V_{1}^{\&}+V_{2}^{\&}+z_{3}\left(a_{3} \cdot \&+a_{4} \theta_{1}+K_{1} u_{1}+d_{12}-\alpha_{2}\right)
\end{aligned}
$$

Design $u_{1}$ as

$$
u_{1}=\left(1 / K_{1}\right)\left(-c_{3} z_{3}-a_{3} \&-a_{4} \theta_{1}-\hat{d}_{12}+a_{r}-a_{2} z_{2}\right)
$$

where $c_{3}$ is a positive control gain, $\alpha_{r}(t)$ can be obtained by using the following filter [34].

$$
\left\{\begin{array}{l}
\phi \&(t)=\vartheta_{1}(t) \\
\vartheta_{1}(t)=-\gamma_{1}\left|\varphi_{1}-\alpha_{2}\right|^{1 / 2} \operatorname{sign}\left(\varphi_{1}-\alpha_{2}\right)+\varphi_{2}(t)(26) \\
\& \&(t)=-\gamma_{2} \operatorname{sign}\left(\varphi_{2}-\vartheta_{1}\right)
\end{array}\right.
$$

Properly choose the parameters $\gamma_{1}, \gamma_{2}$ and let $\alpha_{r}=\varphi_{1}$, then from lemma 5 we have

$$
\alpha_{r}=\varphi_{1}(t)=\alpha_{2}(t), \quad \alpha_{r}=\vartheta_{1}(t)=\alpha_{2}(t)
$$

So the matter of "explosion of complexity" can be work out. Substituting Eq.(25) into Eq.(24) yields

$$
\begin{aligned}
L_{3}^{\&} & \leq-c_{1} z_{1}^{2}-\left(c_{2}-\frac{1}{2}\right) z_{2}^{2}-\left(c_{3}-1\right) z_{3}^{2} \\
& +\frac{1}{2} d_{11}^{6}+\frac{1}{2} d_{12}^{\theta}+\left.\frac{1}{2}\left|\alpha_{r}-\alpha\right|_{2}\right|^{2} \\
& \leq-\lambda_{1} V_{3}+\varepsilon_{1}
\end{aligned}
$$

where $\quad \lambda_{1}=\min \left\{2 c_{1}, 2\left(c_{2}-1 / 2\right) / \bar{M}_{1}, 2\left(c_{3}-1\right)\right\} \quad$ and $\varepsilon_{1}=d_{11}^{B} / 2+d_{12}^{B} / 2+\left|a_{r}-a_{2}\right|^{2} / 2$.

It is clear that $\varepsilon_{1}$ is bounded. Then the closed-loop system with the control law (25) is asymptotically stable. The above discussion can be summarized as the following conclusion.

Theorem 2: By using the control input $u_{1}$ the tracking error $z_{1}$ is bounded and the acceleration is also bounded.

3.2 Robust output restriction controller for slider 2

In this subsection, a robust output restriction controller will be designed to suppress the deformation $\omega(l, t)$ to realize the sync motion of two sliders. By using Eq.(3g) the Eq.(3e) becomes

$$
\begin{aligned}
M_{2}(l, t)= & b_{1} \&(t)+b_{2} \theta_{2}(t)-M_{2}(t) \\
& -f_{2}(t)+E I \omega^{\prime \prime \prime}(l, t)+d_{21}(t)
\end{aligned}
$$

For Eq.(27) define tracking error as

$$
\begin{aligned}
& e_{1}=\omega(l, t)-0=\omega(l, t) \\
& e_{2}=\omega(l, t)-\tau_{1}
\end{aligned}
$$

where $\tau_{1}$ is a virtual control input which will be designed later.

Step 1: Construct the Lyapunov function as

$$
V_{b 1}=\frac{1}{2} \ln \frac{b^{2}}{b^{2}-e_{1}^{2}}
$$

where the target of designing controller is confirm that $|\omega(l, t)|<b, \quad b$ is a positive constant.

The time derivative of $V_{b 1}$ is

$$
V_{b 1}^{\&}=\frac{e_{1}\left(e_{2}+\tau_{1}\right)}{b^{2}-e_{1}^{2}}
$$

where $\tau_{1}=-k_{1} e_{1}-\left(b^{2}-e_{1}^{2}\right) M_{L}, \quad M_{L}=-E I \omega^{\prime \prime \prime}(l, t)$. Substituting $\tau_{1}$ into Eq.(29) yields

$$
L_{b 1}^{\&}=\frac{-k_{1} e_{1}^{2}}{b^{2}-e_{1}^{2}}-e_{1} M_{L}+\frac{e_{1} e_{2}}{b^{2}-e_{1}^{2}}
$$

where $k_{1}$ is a positive control gain.

Step 2: Construct a Lyapunov function $V_{b 2}$ as

$$
V_{b 2}=V_{b 1}+\frac{1}{2} M_{2} e_{2}^{2}
$$

Its time derivative is

$$
\begin{aligned}
V_{b 2}= & I_{b 1}^{\&}+e_{2} M_{2} \& \\
=V_{b 1}^{\&} & +e_{2}\left[b_{1} \&(t)+b_{2} \theta_{2}(t)-M_{2}(t)\right. \\
& \left.-f_{2}(t)+E I \omega^{\prime \prime \prime}(l, t)+d_{21}(t)-M_{2} \&\right]
\end{aligned}
$$

Introduce the virtual control input $\tau_{2}(t)$ and define the tracking error $e_{3}$ as $e_{3}=\theta_{2}(t)-\tau_{2}(t)$, where $\tau_{2}(t)$ is designed as

$$
\begin{gathered}
\tau_{2}=\left(1 / b_{2}\right)\left[-b_{1} \&+f_{2}-E I \omega^{\prime \prime \prime}(l, t)-\hat{d}_{21}+M_{2}\right. \\
\left.+M_{2} \&-k_{2} e_{2}-\frac{\beta e_{2}}{2\left(b^{2}-e_{1}^{2}\right)}-\frac{e_{1}}{b^{2}-e_{1}^{2}}+\frac{\beta k_{1} e_{1}}{b^{2}-e_{1}^{2}}\right]
\end{gathered}
$$

where $k_{2}$ is a positive control gain. 
Substituting Eq.(32) into Eq.(31) yields

$$
\begin{aligned}
L_{b 2}^{\&}=\frac{-k_{1} e_{1}^{2}}{b^{2}-e_{1}^{2}}- & k_{2} e_{2}^{2}-\frac{\beta e_{2}^{2}}{2\left(b^{2}-e_{1}^{2}\right)} \\
& +\frac{\beta k_{1} e_{1} e_{2}}{b^{2}-e_{1}^{2}}-e_{1} M_{L}+b_{2} e_{2} e_{3}+e_{2} d_{21}
\end{aligned}
$$

where $\mathscr{d}_{21}^{0}=d_{21}-\hat{d}_{21}$.

Step 3: Construct the Lypunov function $V_{b 3}$ as

$$
V_{b 3}=V_{b 2}+\frac{1}{2} e_{3}^{2}
$$

Its time derivative is

$$
\begin{aligned}
& V_{b 3}^{\&}=V_{b 2}^{\&}+e_{3} \& \\
& =V_{b 2}^{\&}+e_{3}\left[b_{3} \cdot \underset{z}{\&}(t)+b_{4} \theta_{2}(t)+K_{2} u_{2}(t)+d_{22}(t)-2 \&\right]
\end{aligned}
$$

where $u_{2}$ is designed as

$$
u_{2}=\left(1 / K_{2}\right)\left[-b_{3} \&-b_{4} \theta_{2}-k_{3} e_{3}-b_{2} e_{2}-\hat{d}_{22}+\alpha\right]
$$

where $k_{3}$ is a positive control gain, $\tau_{r}$ can be obtained by the following filter

$$
\left\{\begin{array}{l}
\phi_{1}^{\&}(t)=\vartheta_{2}(t) \\
\vartheta_{2}(t)=-\gamma_{3}\left|\phi_{1}-\tau_{2}\right|^{1 / 2} \operatorname{sign}\left(\phi_{1}-\tau_{2}\right)+\phi_{2}(t) \\
\phi_{2}^{\&}(t)=-\gamma_{4} \operatorname{sign}\left(\phi_{2}-\vartheta_{2}\right)
\end{array}\right.
$$

Choose the parameters $\gamma_{3}, \gamma_{4}$ properly and let $\tau_{r}=\phi_{1}$, then from lemma 5 we have

$$
\underset{r}{\&}=\vartheta_{2}(t)=\&
$$

Substituting Eq.(35) into Eq.(34) yields

$$
\begin{aligned}
& v_{b 3}^{\&}=V_{b 2}^{\&}+e_{3} \& \\
& =\frac{-k_{1} e_{1}^{2}}{b^{2}-e_{1}^{2}}-k_{2} e_{2}^{2}-\frac{\beta e_{2}^{2}}{2\left(b^{2}-e_{1}^{2}\right)}-k_{3} e_{3}^{2}+\frac{\beta k_{1} e_{1} e_{2}}{b^{2}-e_{1}^{2}} \\
& \quad-e_{1} M_{L}+e_{2} d_{21}^{o}+e_{3} d_{22}^{o}+e_{3}(\underset{r}{\&}-\underset{2}{\&})
\end{aligned}
$$

By using Eq.(37) Theorem 3 can be obtained as follows

Theorem 3: Using the boundary control $u_{2}$, the deformation $\omega(x, t)$ of the beam is bounded. Moreover, if the initial value satisfies that $|\omega(l, 0)|<b$, then $\omega(l, t)$ will keep in the area $|\omega(l, t)|<b$.

Proof. Construct the Lyapunov function as

$$
V=V_{b_{3}}+V_{a 1}+V_{a_{2}}+V_{a 3}
$$

where $V_{a 1}=\frac{\beta \rho}{2} \int_{0}^{l} \alpha^{2}(x, t) d x+\frac{\beta E I}{2} \int_{0}^{l}\left[\omega^{\prime \prime}(x, t)\right]^{2} d x$,

$V_{a 2}=\alpha \rho \int_{0}^{l} x \& \&(x, t) \omega^{\prime}(x, t) d x, V_{a 3}=\rho \int_{0}^{l} \omega(x, t) \&(x, t) d x$

$\beta$ and $\alpha$ are positive constants.

From Lemma $7 \quad V_{a 2}$ satisfies the following inequality

$$
\begin{aligned}
\left|V_{a 2}\right| & \leq \alpha \rho l \int_{0}^{l} \alpha^{2}(x, t) d x+\alpha \rho l \int_{0}^{l}\left[\omega^{\prime}(x, t)\right]^{2} d x \\
& \leq \alpha \rho l \int_{0}^{l} c^{2}(x, t) d x+\alpha \rho l^{3} \int_{0}^{l}\left[\omega^{\prime \prime}(x, t)\right]^{2} d x \\
& \leq \chi_{1} V_{a 1}
\end{aligned}
$$

where $\chi_{1}=\max \left(\frac{2 \alpha l}{\beta}, \frac{2 \alpha \rho l^{3}}{\beta E I}\right)$.

From Lemma $7 V_{a 3}$ satisfies that

$$
\begin{aligned}
\left|V_{a 3}\right| & \leq \rho \int_{0}^{l} c^{2}(x, t) d x+\rho \int_{0}^{l} \omega^{2}(x, t) d x \\
& \leq \rho \int_{0}^{l} \&^{2}(x, t) d x+\rho l^{4} \int_{0}^{l}\left[\omega^{\prime \prime}(x, t)\right]^{2} d x \\
& \leq \chi_{2} V_{a 1}
\end{aligned}
$$

where $\chi_{2}=\max \left(\frac{2}{\beta}, \frac{2 \rho l^{4}}{\beta E I}\right)$.

Then we have

$$
0 \leq \kappa_{1}\left(V_{a 1}+V_{b 3}\right) \leq V \leq \kappa_{2}\left(V_{a 1}+V_{b 3}\right)
$$

where $0<\kappa_{1}=1-\chi_{1}-\chi_{2}<1,1<\kappa_{2}=1+\chi_{1}+\chi_{2}<2$. The time derivative of $V_{a 1}(t)$ are as follows:

$$
\begin{aligned}
& V_{a 1}^{\&}=-\beta \rho \int_{0}^{l} \& \&(x, t) d x-\beta E I \&(l, t) \omega^{\prime \prime \prime}(l, t) \\
& \left.\leq \xi_{1} \beta \rho()^{2}+\frac{\beta \rho}{4 \xi_{1}} \int_{0}^{l} \phi^{2}(x, t) d x+\beta \& \& l, t\right) M_{L}
\end{aligned}
$$

where $\xi_{1}$ is a positive constant.

From Eq.(28) and $\tau_{1}$ we further have

$$
\begin{array}{r}
\beta \&(l, t) M_{L}=\beta M_{L}\left[e_{2}-k_{1} e_{1}-\left(b^{2}-e_{1}^{2}\right) M_{L}\right] \\
=\beta \frac{e_{2}^{2}-\alpha^{2}(l, t)-k_{1}^{2} e_{1}^{2}}{2\left(b^{2}-e_{1}^{2}\right)}-\beta \frac{k_{1} \&(l, t) e_{1}}{b^{2}-e_{1}^{2}} \\
-\frac{\beta}{2}\left(b^{2}-e_{1}^{2}\right) M_{L}^{2}-\beta k_{1} e_{1} M_{L}
\end{array}
$$

Substituting Eq.(42) into Eq.(41) yields

$$
\begin{gathered}
\left.L_{a 1}^{\&} \leq \xi_{1} \beta \rho()^{2}+\frac{\beta \rho}{4 \xi_{1}} \int_{0}^{l} \&^{2}(x, t) d x+\beta c \& l, t\right) M_{L} \\
\leq \xi_{1} \beta \rho()^{2}+\frac{\beta \rho}{4 \xi_{1}} \int_{0}^{l} \&^{2}(x, t) d x+\beta \frac{e_{2}^{2}-c^{2}(l, t)-k_{1}^{2} e_{1}^{2}}{2\left(b^{2}-e_{1}^{2}\right)} \\
-\beta \frac{\left.k_{1} \& \& l, t\right) e_{1}}{b^{2}-e_{1}^{2}}-\frac{\beta}{2}\left(b^{2}-e_{1}^{2}\right) M_{L}^{2}-\beta k_{1} e_{1} M_{L}
\end{gathered}
$$

The time derivative of $V_{a 2}$ is as follows:

$$
\begin{aligned}
& \left.L_{a 2}^{\&}=\alpha \rho \int_{0}^{l} x \& x, t\right) \omega^{\prime}(x, t) d x+\alpha \rho \int_{0}^{l} x d \&(x, t) \&^{2}(x, t) d x \\
& =-\alpha \rho \int_{0}^{l} \omega^{\prime}(x, t) x d x-\alpha E I \int_{0}^{l} x \omega^{\prime \prime \prime \prime}(x, t) \omega^{\prime}(x, t) d x \\
& +\alpha \rho \int_{0}^{l} x c \&(x, t) \& \&(x, t) d x \\
& =A_{1}+A_{2}+A_{3}
\end{aligned}
$$

where

$$
\begin{aligned}
& A_{1}=-\alpha \rho \int_{0}^{l} \omega^{\prime}(x, t) x d x, \\
& A_{2}=-\alpha E I \int_{0}^{l} x \omega^{\prime \prime \prime \prime}(x, t) \omega^{\prime}(x, t) d x, \\
& A_{3}=\alpha \rho \int_{0}^{l} x \&(x, t) \&(x, t) d x .
\end{aligned}
$$

From Lemma 7 it is clear that $A_{1}$ satisfies that

$$
A_{1} \leq \alpha(\rho l+\rho)+\alpha(\rho l+\rho) l^{4} \int_{0}^{l}\left[\omega^{\prime \prime}(x, t)\right]^{2} d x(44)
$$

By using the integration by parts the following inequality for $A_{2}$ can be obtained

$$
A_{2} \leq\left(\alpha l^{2}-\frac{3}{2} \alpha E I\right) \int_{0}^{l}\left[\omega^{\prime \prime}(x, t)\right]^{2} d x+\alpha l M_{L}^{2}
$$

$A_{3}$ can be converted into the following form by using 
the integration by parts

$$
\left.A_{3}=\frac{1}{2} \alpha \rho l[\& \& l, t)\right]^{2}-\frac{1}{2} \alpha \rho \int_{0}^{l} \alpha^{2}(x, t) d x
$$

With employment of inequalities (44)-(46) $I_{a 2}^{\&}$ becomes

$$
\begin{aligned}
\mathbb{V}_{a 2}^{\&} \leq & {\left[\alpha(\rho l+\rho) l^{4}+\alpha l^{2}-\frac{3}{2} \alpha E I\right] \int_{0}^{l}\left[\omega^{\prime \prime}(x, t)\right]^{2} d x+\alpha l M_{L}^{2} } \\
& \left.-\frac{1}{2} \alpha \rho \int_{0}^{l} \alpha^{2}(x, t) d x+\alpha(\rho l+\rho)+\frac{1}{2} \alpha \rho l[\alpha \& l, t)\right]^{2}
\end{aligned}
$$

The time derivative of $V_{a 3}$ satisfies that

$$
\begin{array}{r}
l_{a 3}^{\&} \leq-E I \int_{0}^{l}\left[\omega^{\prime \prime}(x, t)\right]^{2} d x+\rho \int_{0}^{l} c^{2}(x, t) d x+ \\
e_{1} M_{L}+\rho+l^{4} \rho \int_{0}^{l}\left[\omega^{\prime \prime}(x, t)\right]^{2} d x
\end{array}
$$

The Eq.(37) can be further converted into

$$
\begin{aligned}
& L_{b 3}^{\&} \leq \frac{-k_{1} e_{1}^{2}}{b^{2}-e_{1}^{2}}-k_{2} e_{2}^{2}-\frac{\beta e_{2}^{2}}{2\left(b^{2}-e_{1}^{2}\right)}-k_{3} e_{3}^{2} \\
& +\frac{\beta k_{1} e_{1} e_{2}}{b^{2}-e_{1}^{2}}-e_{1} M_{L}+e_{2} d_{21}^{o}+e_{3} d_{22}+e_{3}(d-2) \\
& \leq \frac{-k_{1} e_{1}^{2}}{b^{2}-e_{1}^{2}}-\left(k_{2}-1\right) e_{2}^{2}-\frac{\beta e_{2}^{2}}{2\left(b^{2}-e_{1}^{2}\right)}-\left(k_{3}-2\right) e_{3}^{2}
\end{aligned}
$$

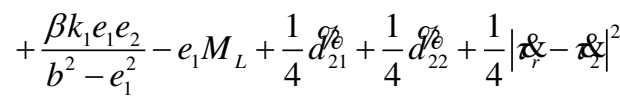

Combining inequalities (43), (47)-(49) we have

$$
\begin{aligned}
& I \& \leq-\left(k_{1}-\frac{\beta k_{1}^{2}}{2}\right) \frac{e_{1}^{2}}{b^{2}-e_{1}^{2}}-\left(k_{2}-1\right) e_{2}^{2}-\left(k_{3}-2\right) e_{3}^{2} \\
& -\left[\frac{3}{2} \alpha E I+E I-\alpha(\rho l+\rho) l^{4}-\alpha l^{2}-\rho l^{4}\right] \int_{0}^{l}\left[\omega^{\prime \prime}(x, t)\right]^{2} d x \\
& -\left[\frac{1}{2} \alpha \rho-\frac{\beta \rho}{4 \xi_{1}}-\rho\right] \int_{0}^{l} \& \&(x, t) d x-\frac{\beta}{2}\left(b^{2}-e_{1}^{2}\right) M_{L}^{2} \\
& -\left[\frac{\beta}{2\left(b^{2}-e_{1}^{2}\right)}-\frac{1}{2} \alpha \rho l\right] \&^{2}(l, t)+\left[\xi_{1} \beta \rho+\alpha(\rho l+\rho)+\rho\right] \\
& +\alpha l M_{L}^{2}+\frac{1}{4} d_{21}^{\delta}+\frac{1}{4} d_{22}^{\delta}+\frac{1}{4}|\alpha \underset{r}{\alpha}-\alpha|^{2}
\end{aligned}
$$

where the positive control gains are selected to satisfy that

$$
\left\{\begin{array}{l}
\mu_{1}=\left(k_{1}-\frac{\beta k_{1}^{2}}{2}\right)>0, \quad \mu_{2}=k_{2}-1>0 \\
\mu_{3}=k_{3}-2>0, \mu_{4}=\frac{1}{2} \alpha \rho-\frac{\beta \rho}{4 \xi_{1}}-\rho>0, \\
\mu_{5}=\frac{3}{2} \alpha E I+E I-\alpha(\rho l+\rho) l^{4}-\alpha l^{2}-\rho l^{4}>0 \\
\mu_{6}=\frac{\beta}{2 b^{2}}-\frac{1}{2} \alpha \rho l>0
\end{array}\right.
$$

According to Lemma 2 in [36], we have

$$
\begin{aligned}
V^{\&} & \leq-\mu_{1} \ln \frac{e_{1}^{2}}{b^{2}-e_{1}^{2}}-\mu_{2} e_{2}^{2}-\mu_{3} e_{3}^{2}-\mu_{6} \&^{2}(l, t) \\
& -\mu_{4} \int_{0}^{l} \&^{2}(x, t) d x-\mu_{5} \int_{0}^{l}\left[\omega^{\prime \prime}(x, t)\right]^{2} d x+\varepsilon_{2} \\
& \leq-\lambda_{3}\left(V_{a 1}+V_{b 3}\right)+\varepsilon_{2}
\end{aligned}
$$

where

$$
\begin{aligned}
& \lambda_{3}=\min \left\{2 \mu_{1}, 2 \mu_{2} / M_{2}, 2 \mu_{3}, 2 \mu_{4} / \beta \rho, 2 \mu_{5} / \beta E I\right\} \text {, } \\
& \varepsilon_{2}=\left[\xi_{1} \beta \rho+\alpha(\rho l+\rho)+\rho\right]
\end{aligned}
$$

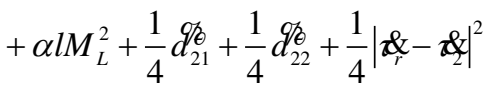

From Theorem 1 and Assumption 2 it is clear that $\varepsilon_{2}$ is bounded and assume that $\varepsilon_{2}$ satisfies $\varepsilon_{2} \leq \bar{\varepsilon}_{2}$, where $\bar{\varepsilon}_{2}$ is a positive constant.

Combining Eq.(40) and (50) we have

$$
V^{\&} \leq-\lambda_{4} V+\bar{\varepsilon}_{2}
$$

where $\lambda_{4}=\lambda_{3} / \kappa_{2}$.

Multiplying $e^{\lambda_{4} t}$ on both sides of Eq.(51) yields

$$
\frac{\partial}{\partial t}\left(V(t) e^{\lambda_{4} t}\right) \leq \bar{\varepsilon}_{2} e^{\lambda_{4} t}
$$

Integrating the inequality (52) with respect to $t$ results in

$$
\begin{aligned}
V(t) & \leq V(0) e^{-\lambda_{4} t}+\frac{\bar{\varepsilon}_{2}}{\lambda_{4}}\left(1-e^{-\lambda_{4} t}\right) \\
& \leq V(0) e^{-\lambda_{4} t}+\bar{\varepsilon}_{2} / \lambda_{4}
\end{aligned}
$$

Then we have a conclusion that $V(t)$ is bounded. Next $\omega(x, t)$ is bounded will be shown.

From Lemma 6 we obtain that

$$
\frac{1}{l} \omega(x, t) \leq \int_{0}^{l}\left[\omega^{\prime}(x, t)\right]^{2} d x
$$

By using Lemma 7 we further have

$$
\frac{\beta E I}{2 l^{3}} \omega^{2}(x, t) \leq \frac{\beta E I}{2} \int_{0}^{l}\left[\omega^{\prime \prime}(x, t)\right]^{2} d x \leq V_{a 1} \leq \frac{V(t)}{\kappa_{1}}(54)
$$

Then from inequality (53) we can get that

$$
|\omega(x, t)| \leq \sqrt{\frac{2 l^{3}}{\beta E I \kappa_{1}}\left[V(0) e^{-\lambda_{4} t}+\frac{\bar{\varepsilon}_{2}}{\lambda_{4}}\right]}
$$

where $x \in[0, l]$.

From inequalities (38) and (39), we have $V_{a 1}+V_{a 2}+V_{a 3}$ is bounded. Moreover, the term $V_{b 3}$ satisfies that $V_{b 3} \rightarrow \infty$, as $|\omega(l, t)| \rightarrow b$. Then from the Lemma 1 presented in [37] we can get that if $|\omega(l, 0)|<b$, the deformation $\omega(l, t)$ will keep in the area $|\omega(l, t)|<b$.

The proof is completed.

Therefore, based on the Theorem 2 and 3 we have the two sliders can track the desired trajectory while keep synchronous movement.

\section{Simulation results}

In order to illustrate the system performance, an assumed modes method will be used to find the approximative solution of the closed-loop system. Since the higher-order modes have little influence on the deformation of the beam, only the first ten assumed modes of the beam are used in the simulations. For more simulation details readers can refer to the reference [11]. The parameters of two sliders and the beam are shown in Table 1.

Table.1 Simulation parameters 


\begin{tabular}{ll}
\hline Parameter & Value \\
$\rho$ & $20 \mathrm{~kg} / \mathrm{m}$ \\
$E I$ & $500 \mathrm{~N} \bullet \mathrm{m}^{2}$ \\
$l$ & $1 \mathrm{~m}$ \\
$M_{1}$ & $30 \mathrm{~kg}$ \\
$M_{2}$ & $20 \mathrm{~kg}$ \\
\hline
\end{tabular}

The initial position of slider 1 is set to be $x_{1}(0)=1 \mathrm{~m}$ and the initial position of slider 2 is set to be $x_{2}(0)=1.015 \mathrm{~m}$. Since the two sliders are restrained by the flexible beam, they are very close to each other. The desired trajectory are selected as $x_{d}=\sin (2 t)$. The model parameters in Eq.(3) are selected as $a_{1}=b_{1}=100, a_{2}=b_{2}=1, \quad a_{3}=10, a_{4}=2$, $K_{1}=10, b_{3}=8 \quad, \quad b_{4}=3 \quad, \quad K_{2}=5, d_{11}=$ $250 \sin (2 t) \quad, \quad d_{12}=100 \sin (4 t) \quad, \quad d_{21}=130 \sin (3 t)+$ $120 \sin (t)$ and $d_{22}=150 \sin (5 t)$. The design parameters for observers can be selected according to the following remark.

Remark 3: The observer parameters in slider 2 are the same as those in slider 1 . So only the parameters in slider 1 are given. The design parameters of observers should be selected to satisfy Eq. (15) at first. $\tau_{1}$ and $\tau_{2}$ are the time constant of the low-pass filter in Eq.(13) and (14). A small $\tau_{1}$ or $\tau_{2}$ can obtain fast approximation of the input signal. So $\tau_{1}$ and $\tau_{2}$ are both selected as 0.01. $\delta_{10}$ and $\delta_{20}$ are also selected to be small for forcing the system to the safety margin faster but they should be larger than the amplitude of computational error. In the simulation $\delta_{10}$ and $\delta_{20}$ are selected as 0.8. $\beta_{1}$ and $\beta_{2}$ should be large enough to dominate the effects of $L_{11}$ and $L_{12}$, where $L_{11}$ and $L_{12}$ do not need to be known exactly. $\alpha_{1}$ and $\alpha_{2}$ are selected to be 0.99 to reduce the effects of $L_{11}$ and $L_{12} . \beta_{1}$ and $\beta_{2}$ are both selected as $1 \times 10^{5}$. $c_{1}, c_{2}, k_{10}$ and $k_{20}$ are safety factors. In the simulations $c_{1}=10, c_{2}=8, k_{10}=k_{20}=0.1 . r_{10}$ and $r_{20}$ are used to regulate the estimation errors of the absolute value of disturbances. They are selected as 10 (see Fig.17). In addition the matrix $V_{1}$ and gain $S_{1}$ are designed as

$$
V_{1}=\left[\begin{array}{cc}
-2.5 & 0.005 \\
2.5 & -0.006
\end{array}\right], \quad S_{1}=\left[\begin{array}{c}
-22.5 \\
-4810
\end{array}\right] \text {. }
$$

The control gains of the proposed controller (25) and (35) are selected as $c_{1}=c_{2}=100, c_{3}=30, \gamma_{1}=120$, $\gamma_{2}=10, k_{1}=0.18, k_{2}=100, k_{3}=30, \quad \gamma_{3}=150$, $\gamma_{4}=10, \beta=11$ and $\alpha=4$. The constrain area $b$ is set to 0.03 .

The sync motion performance of the closed-loop system is shown in Fig.2-8, where Fig.2-4 display the performance without disturbance compensation and
Fig.5-8 illustrate the performance with the proposed disturbance compensation. From Fig. 5 it is shown that the proposed controller can track the desired trajectory while keeping sync motion. With employment of the proposed disturbance compensation the sync errors can be suppressed to be smaller than those without the disturbance compensation from the comparison of Fig.2 and Fig.6. The corresponding shear forces are shown in Fig.3 and Fig.7, respectively. Fig.4 and Fig.8 illustrate the deformation of the beam from $t=0$ to $10 \mathrm{~s}$. It is clear that the deformation in Fig. 8 is smaller than that in Fig.4. The control inputs of two sliders are shown in Fig. 21 and 22 respectively.

The performance of the proposed disturbance observers are displayed in Fig.9-20, where Fig.9-14 illustrate the performance of observers in slider 1 and Fig.15-20 show the performance of observers in slider 2 . From Fig.9-10 and 13-14 it can be obtained that the external disturbance can be estimated effectively by the proposed estimators. But the fixed gains method proposed in [27] may lead to serious chattering when the upper bound of external disturbance cannot be known exactly. When $d_{T}$ and $\bar{d}_{T}$ in Eq.(16) are chosen as $[-20,-500]$ and $[20,500]$, the estimation of disturbance is shown in Fig.13 and 14. It is clear that the chattering phenomenon is serious. However, the proposed variable gain method can solve this problem. $d_{T}$ and $\bar{d}_{T}$ can be replaced by the estimation of upper bound of the external disturbances. From Fig. 9 and 10 it is clear that the chattering phenomenon can be greatly weakened. The estimation of upper bound of external disturbances are shown in Fig.11 and 12. The fixed gain method in [27] displays the same issue in control channel 2 from Fig.19 and 20. And it can be solved by the proposed method from Fig.15 and 16. The estimations of upper bound of the external disturbances in slider 2 are shown in Fig.17 and 18. The estimation error is $r_{10}=r_{20}=10$.

\section{Conclusion}

This paper proposes a robust sync motion control method to realize the sync tracking control of two sliders which are restricted by a flexible beam. The system models are given at first. In the models the dynamics of actuators are also considered. With employment of the proposed models a novel variable gain sliding mode interval observer is designed to estimate the external disturbance while reducing the chattering phenomenon. Then an output restriction boundary controller is designed to restrict the deformation of the beam. With the help of the controller close-loop system is proved to be asymptotically stable by using Lyapunov direct method. Finally, numerical simulations illustrate that the proposed method is effective compared with the existing methods. 


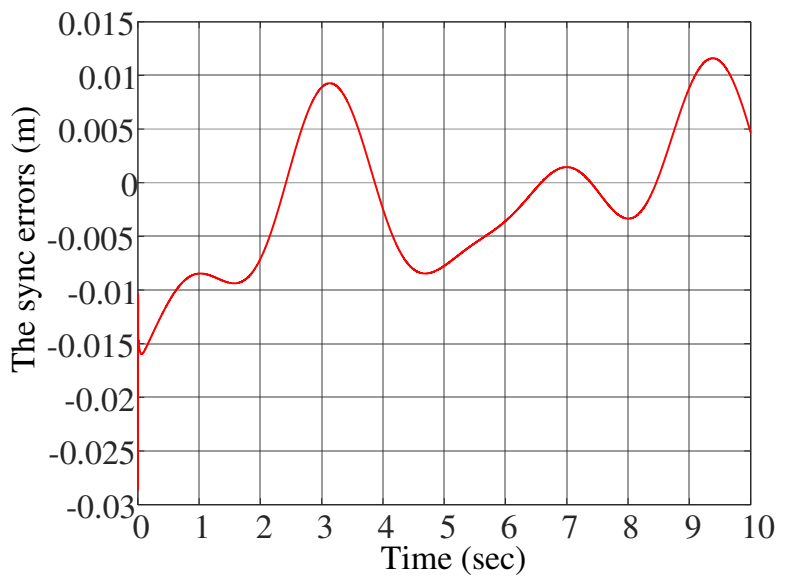

Fig. 2 The sync errors of two sliders without disturbance estimation

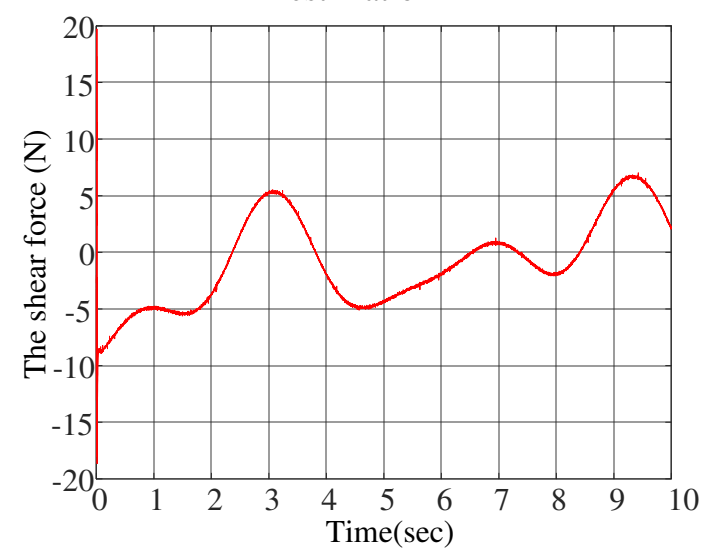

Fig.3 The shear force of the beam without disturbance estimation

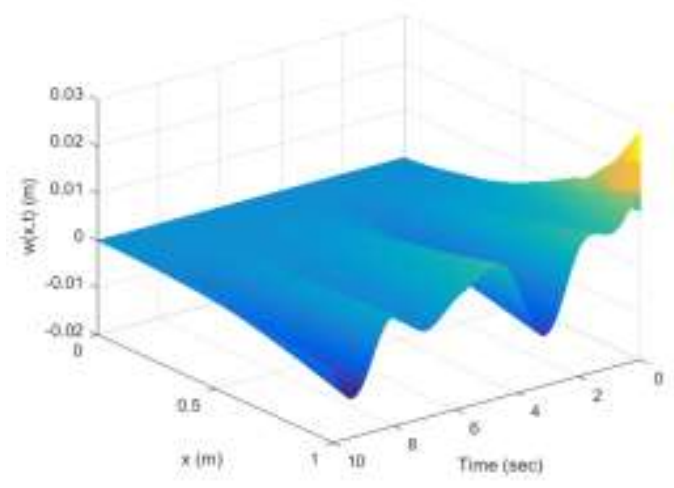

Fig.4 The deformation of the beam without disturbance

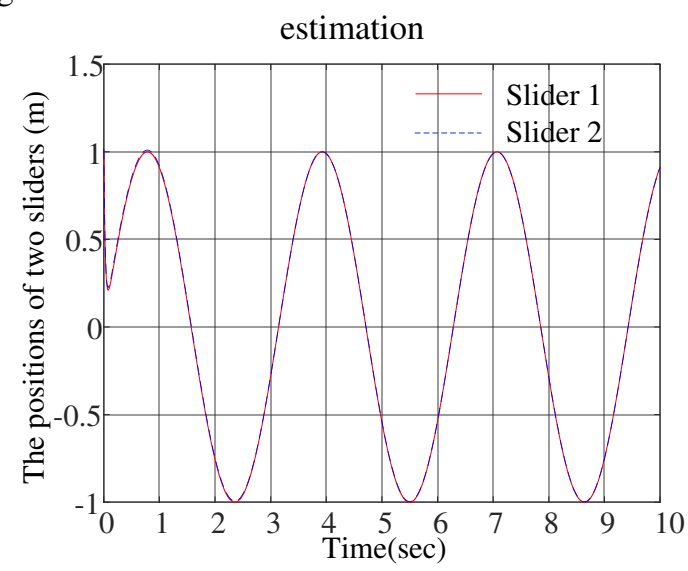

Fig.5 The positions of two sliders with disturbance

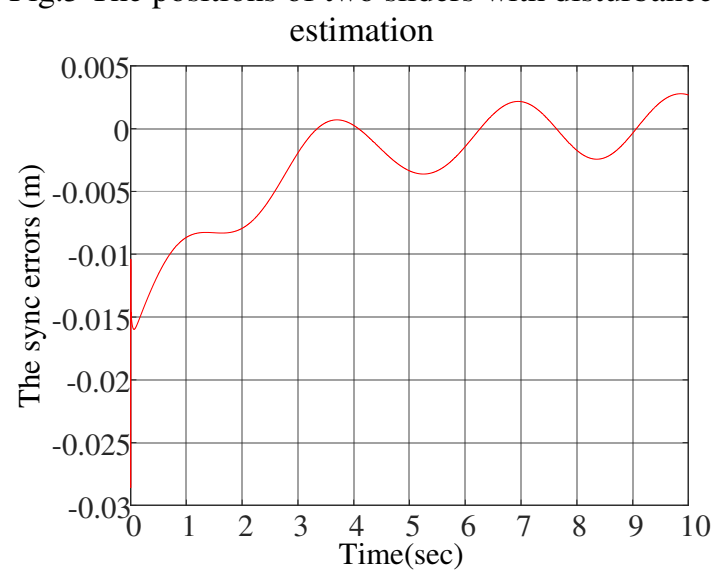

Fig.6 The sync errors of two sliders with disturbance estimation

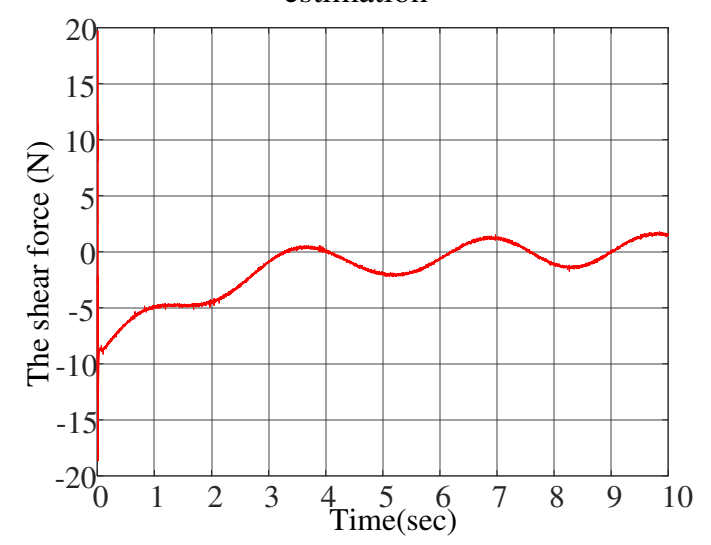

Fig.7 The shear force of the beam with disturbance estimation

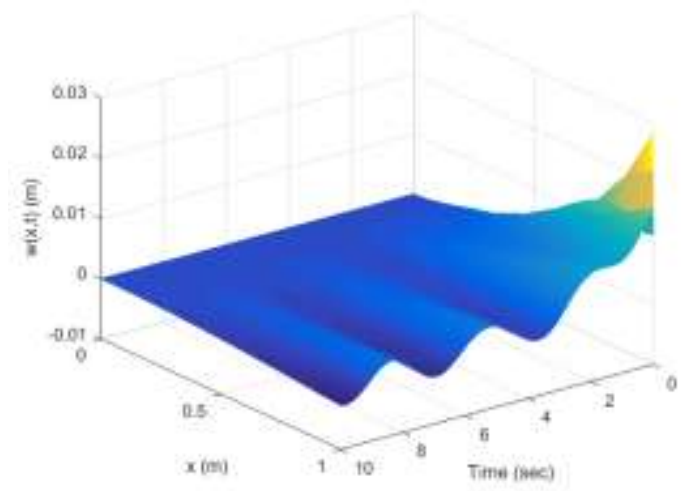

Fig. 8 The deformation of the beam with disturbance estimation 


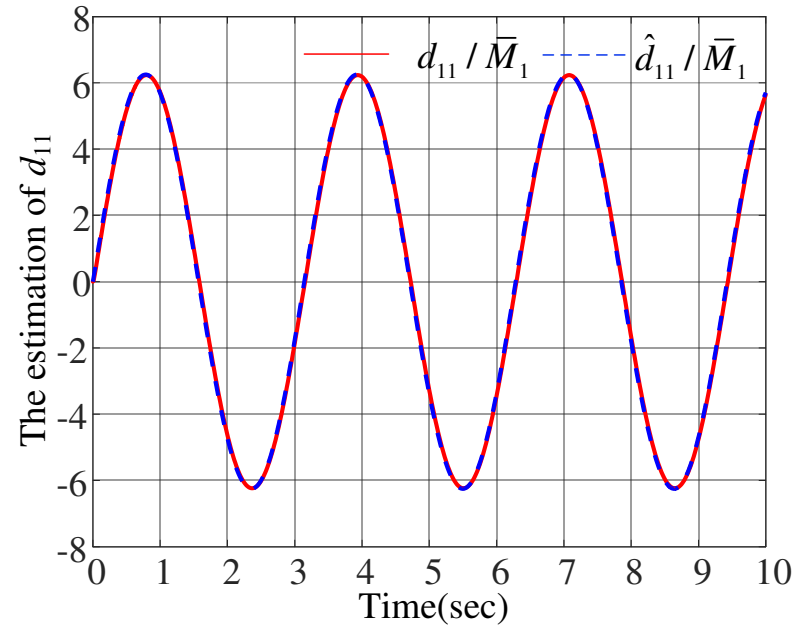

Fig.9 The estimation of $d_{11}$ with the proposed method

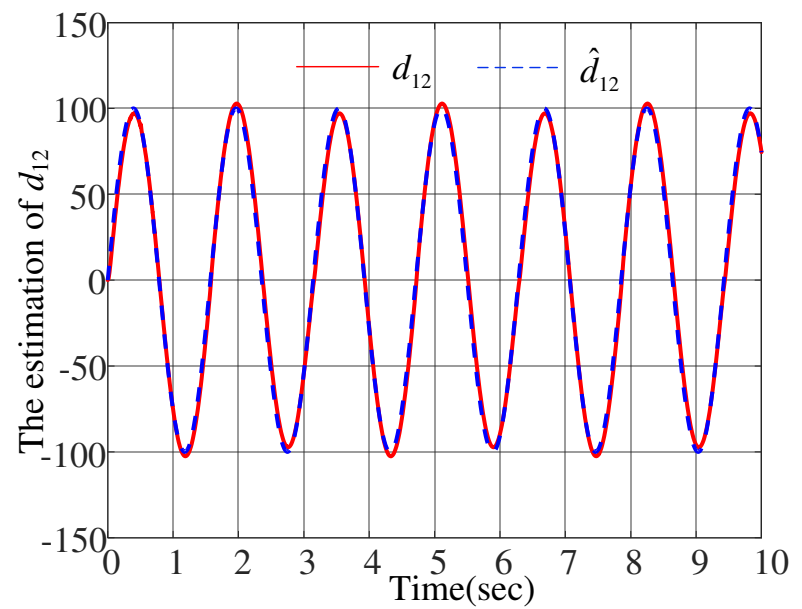

Fig.10 The estimation of $d_{12}$ with the proposed method

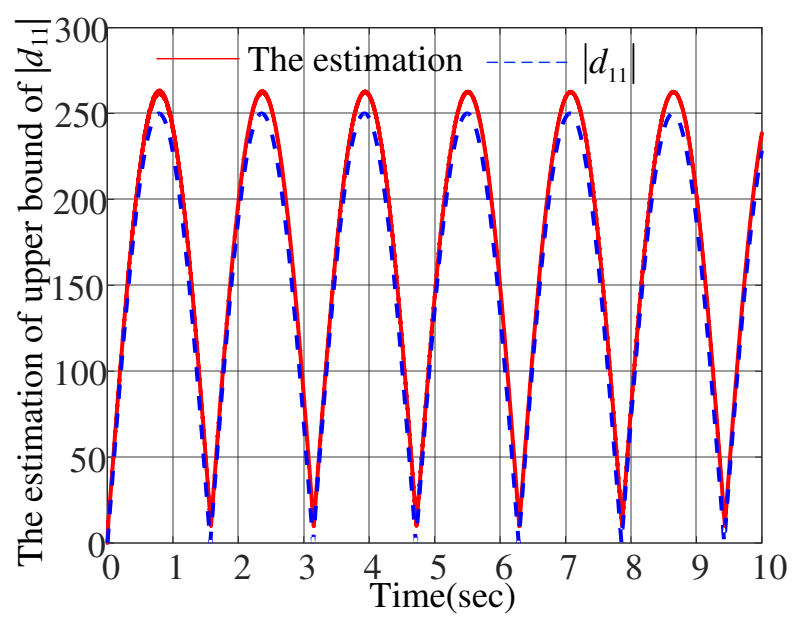

Fig.11 The estimation of upper bound of $\left|d_{11}\right|$

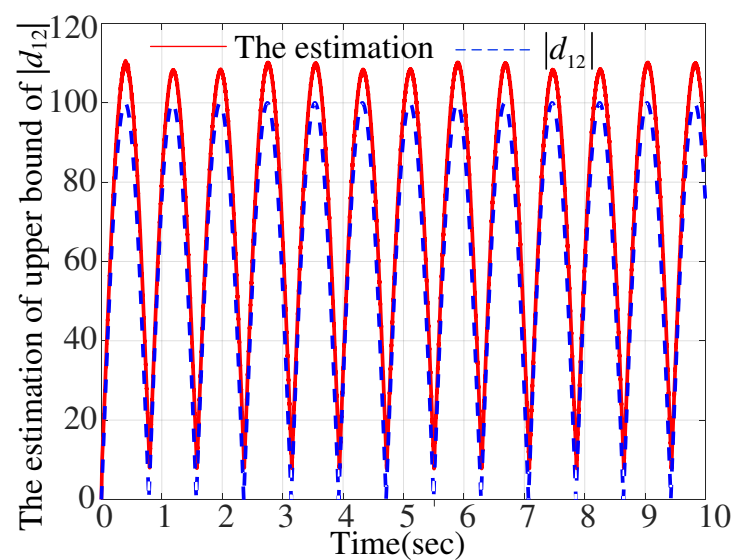

Fig.12 The estimation of upper bound of $\left|d_{12}\right|$

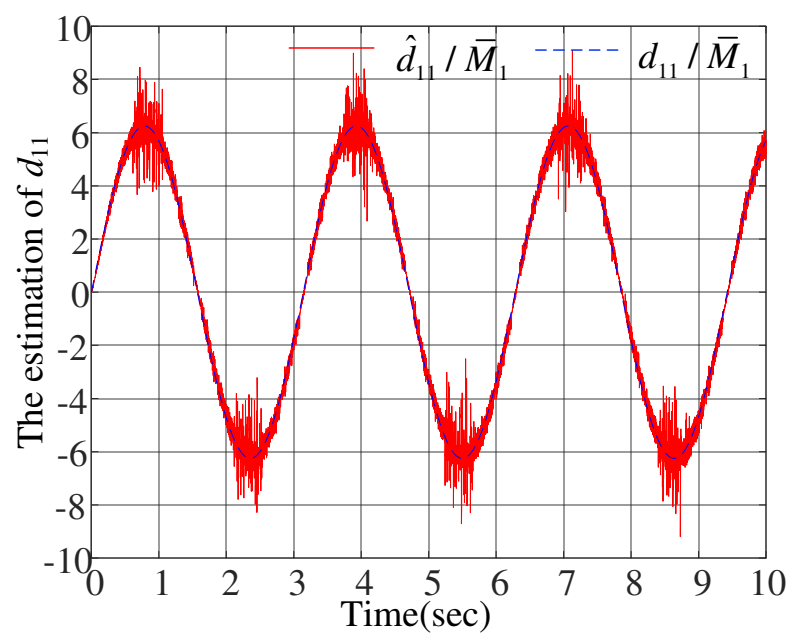

Fig.13 The estimation of $d_{11}$ with fixed gain in [27]

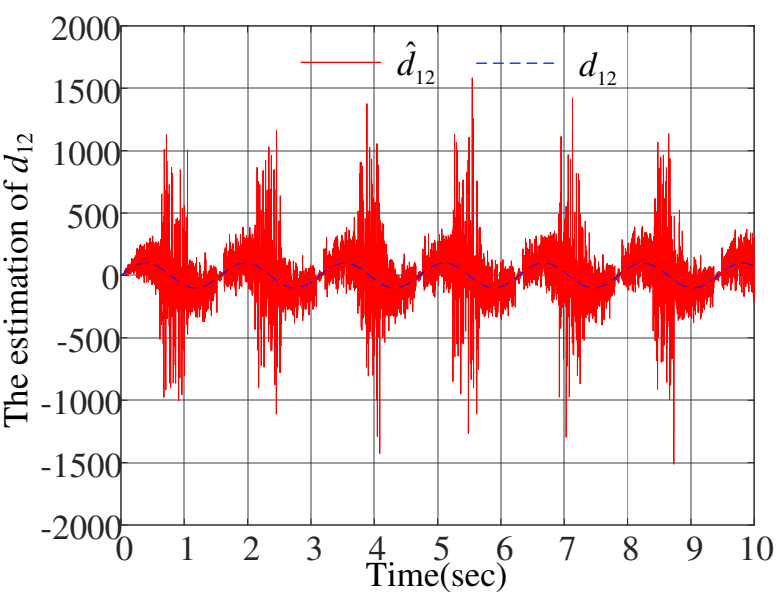

Fig.14 The estimation of $d_{12}$ with fixed gain in [27] 


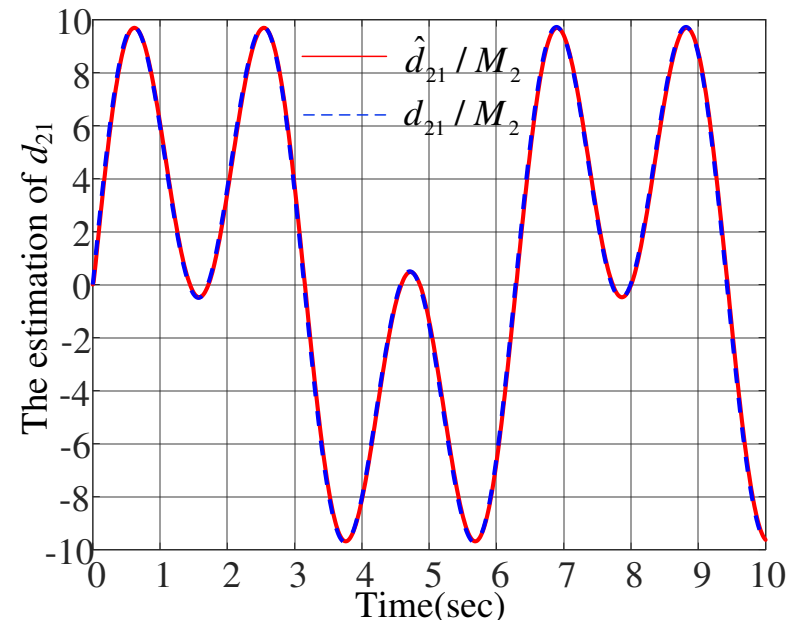

Fig.15 The estimation of $d_{21}$ with the proposed method

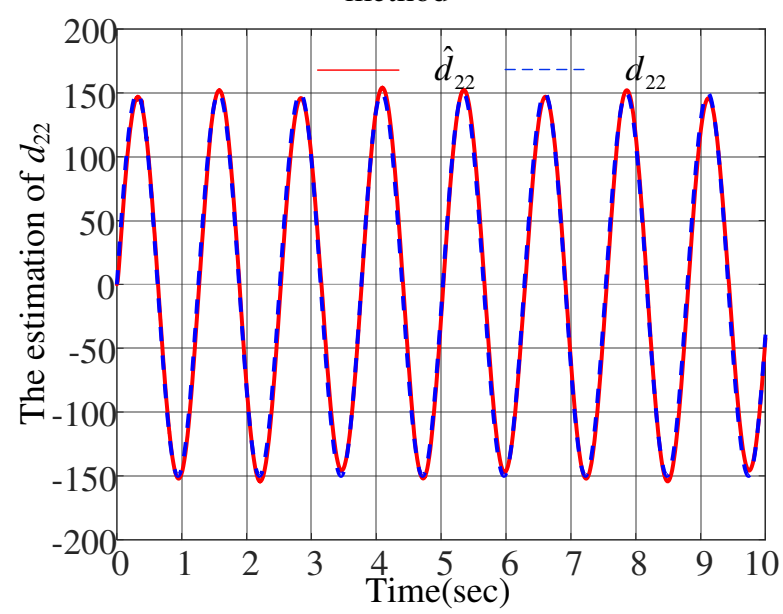

Fig.16 The estimation of $d_{22}$ with the proposed

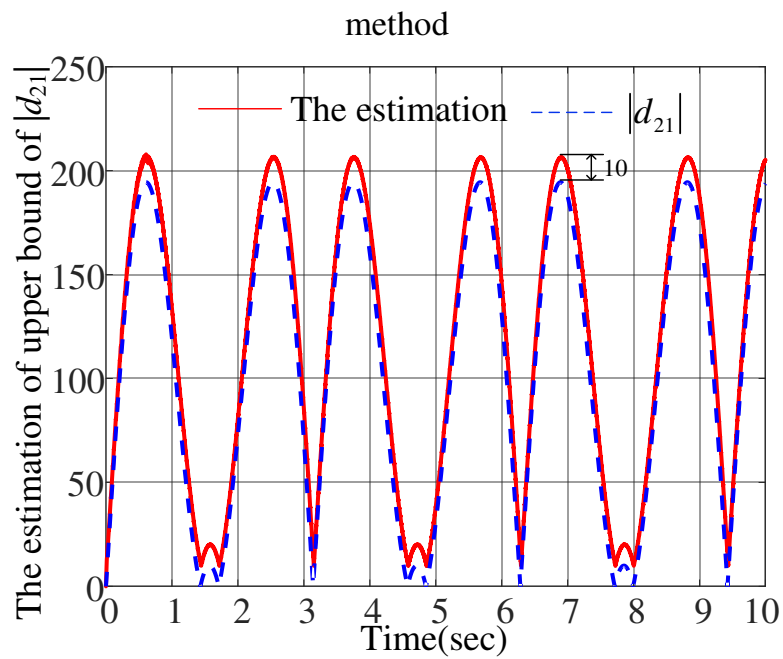

Fig.17 The estimation of upper bound of $\left|d_{21}\right|$

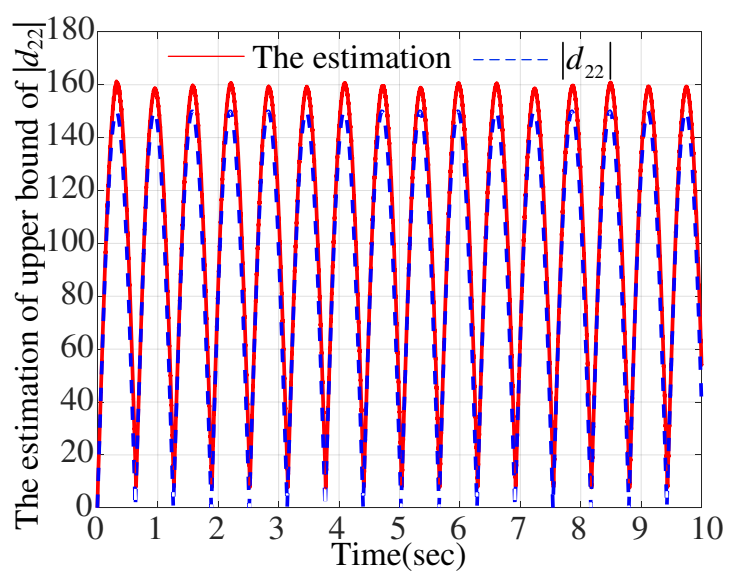

Fig.18 The estimation of upper bound of $\left|d_{22}\right|$

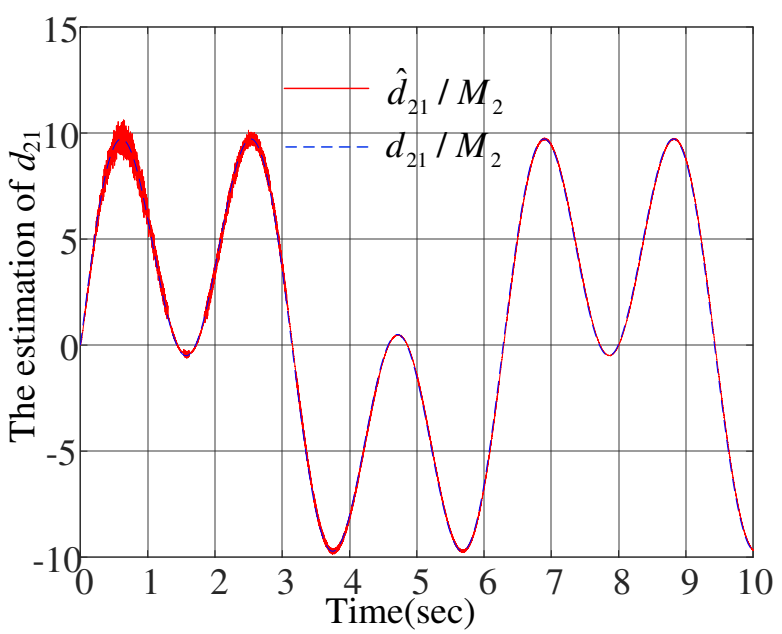

Fig.19 The estimation of $d_{21}$ with fixed gain in [27]

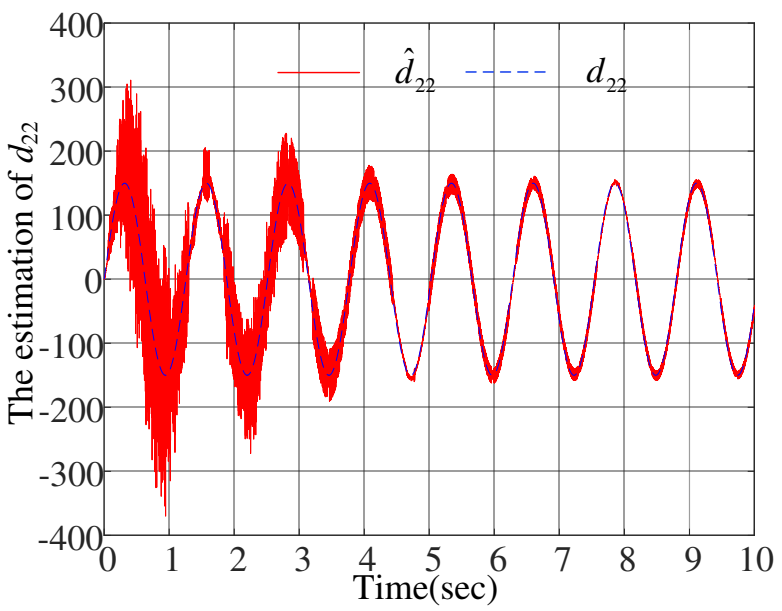

Fig.20 The estimation of $d_{22}$ with fixed gain in [27] 


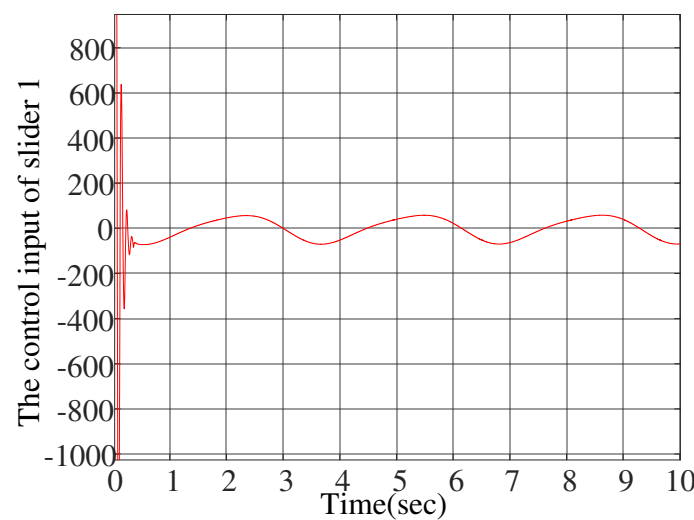

Fig. 21 The control input of slider 1

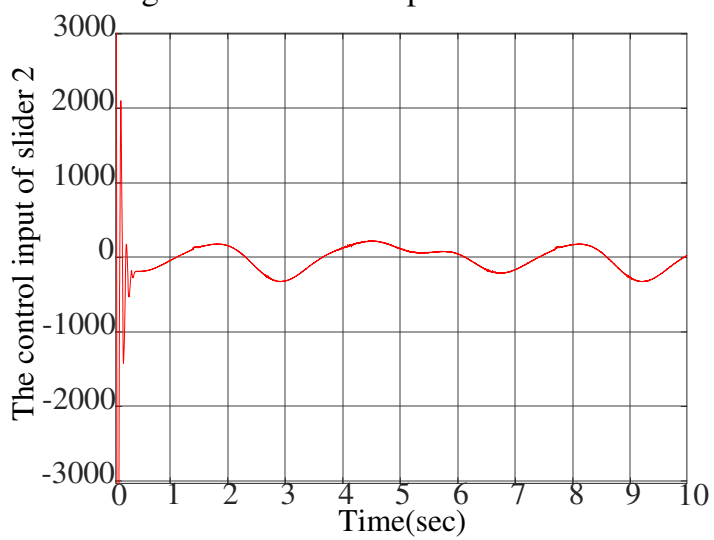

Fig.22 The control input of slider 2

\section{Conflict of Interest:}

I declare that this paper has no conflict of interest.

\section{Data availability statement:}

The conclusions of this paper are all illustrated in the figures. Source data for figures are provided with the paper. I declare that one can obtain these data from me on reasonable request.

\section{Reference}

[1]Weiwei Shang, Bingyuan Zhang, Bin Zhang et al. Synchronization control in the cable space for cable-driven parallel robots. IEEE Transactions on Industrial Electronics, 2018, 66(6): 4544-4554.

[2]Huaiyuan Jia, Weiwei Shang, Fei Xie et al. Second-order sliding-mode-based synchronization control of cable-driven paralle robots. IEEE/ASME Transactions on Mechatronics, 2020, 25(1):383-394.

[3]Quan Vinh Doan, Tien Dung Le and Anh Tuan Vo. Synchronization Full-oder Terminal Sliding Mode Control for an Uncertain 3-DOF Planar Parallel Robotic Manipulator. Applied Sciences, 2019, 9(9):1756-1772.

[4]Cong Li, Bin Yao and Qingfeng Wang. Modeling and Synchronization Control of a Dual Drive Industrial Gantry Stage. IEEE/ASME Transactions on Mechatronics, 2018, 23(6):2940-2951.

[5]Cong Li, Chao Li, Zheng Chen et al. Adaptive thrust allocation based synchronization control of a dual drive gantry stage. Mechatronics, 2018, 54:68-77.

[6]Zheng Chen, Chao Li, Bin Yao et al. Integrated Coordinated/Synchronized Contouring control of a dual linear motor driven gantry. IEEE Transactions on Industrial Electronics, 2020, 5(67): 3944-3954.

[7] Cun Shi, Shaoping Wang, Xingjian Wang et
al.Active fault-tolerant control of dissimilar redundant actuation system based on performance degradation reference models. Journal of the Franklin Institute, 2016, 354(2): 1087-1108.

[8] Waheed Ur Rehman, Wang Shaoping, Wang Xingjian, Fan Lei, Kamran Ali Shah. Motion synchronization in a dual redundant HA/EHA system by using a hybrid integrated intelligent control design. Chinese Journal of Aeronautics, 2016, 29(3): 789-798.

[9] Cun Shi, Xingjian Wang, Shaoping Wang, et al. Adaptive decoupling synchronous control of dissimilar redundant actuation system for large civil aircraft. Aerospace Science and Technology, 2015, 47:114-124. [10] Haibin Dou, Shaoping Wang. Robust adaptive motion/force control for motion synchronization of multiple uncertain two-link manipulators. Mechanism and Machine Theory. 2013, 67:77-93.

[11] Haibin, Dou, Shaoping Wang. A boundary control for motion synchronization of a two-manipulator system with a flexible beam. Automatica, 2014, 50: 3088-3099.

[12]Dongya Zhao, Quanmin Zhu. Position synchronised control of multiple robotic manipulators based on integral sliding mode. International Journal of systems science. 2014, 45:556-570.

[13] Ting Li, Ting Yang, Yuyan Cao et al. Disturbance-Estimation Based Adaptive Backstepping Fault-Tolerant Synchronization Control for a Dual Redundant Hydraulic Actuation System With Internal Leakage Faults. IEEE Access, 2019, 7:73106-73119.

[14]Haibin Dou. Adaptive robust fault-tolerant control for a two-slider synchronization system with a flexible beam. Journal of the Franklin Institute, 2021, 358: 9561-9586

[15] Ting Li, Ting Yang, Yuyan Cao et al. Adaptive robust fault-tolerant synchronization control for a dual redundant hydraulic actuation system with common-mode fault. Mathematical Problems in Engineering, 2018, 2018:1-14.

[16] Salman Ijza, Mirza Tariq Hamayun, Lin Yan et al. Adaptive fault tolerant control of dissimilar redundant actuation system of civil aircraft based on integral sliding mode control strategy. Transactions of the institute of measurement and control, 2019, 41(1) :1-13. [17]Feng-Fei Jin and Bao-Zhu Guo. Lyapunov approach to output feedback stabilization for the Euler-Bernoulli beam equation with boundary input disturbance. Automatica, 2015, 52:95-102.

[18] Bao-Zhu Guo and Wen Kang. Lyapunov approach to the boundary stabilisation of a beam equation with boundary disturbance. International journal of control, 2013, 87(5):925-939.

[19] Bao-Zhu Guo and Feng-Fei Jin. The active disturbance rejection and sliding mode control approach to the stabilization of the Euler-Bernoulli beam equation with boundary input disturbance. Automatica, 2013, 49(9): 2911-2918.

[20] Bao-Zhu Guo and Jun-Jun Liu. Sliding mode control and active disturbance rejection control to the stabilization of one-dimensional Schrödinger equation 
subject to boundary control matched disturbance. International journal of robust and nonlinear control, 2013, 24(16):2194-2212.

[21] Hongyinping Feng and Bao-Zhu Guo. A New Active Disturbance Rejection Control to Output Feedback Stabilization for a One-Dimensional Anti-Stable Wave Equation With Disturbance. IEEE Transactions on Automatic Control, 2017, 62(8): 3774-3787.

[22] Tingting Jiang, Jinkun Liu, and Wei He. Boundary control for a flexible manipulator based on infinite dimensional disturbance observer. Journal of sound and Vibration, 2015, 348:1-14.

[23]Fang Guo, Yu Liu, Yilin Wu, Fei Luo. Observer-based backstepping boundary control for a flexible riser system. Mechanical Systems and Signal Processing, 2018, 111:314-330.

[24] Zhijia Zhao, Xiuyu He and Choon Ki Ahn. Boundary disturbance observer-Based control of a vibrating single-link flexible manipulator. IEEE Transactions on Systems, Man, and Cybernetics: System, 2021, 51(4):2382-2390.

[25] Zhijia Zhao and Zhijie Liu. Finite-Time Convergence Disturbance Rejection Control for a Flexible Timoshenko Manipulator. IEEE/CAA Journal of Automatica Sinica, 2021, 8(1):157-168.

[26]D. Efimov and T. Raissi. Design of interval observers for uncertain dynamical systems. Automation and Remote Control, 2016, 77:191-225.

[27]Xianghua Wang, Chee Pin Tan, Lanjun Liu et al. A novel unknown input interval observer for systems not satisfying relative degree condition. International Journal of Robust and Nonlinear Control, 2021, 31(7): 2762-2782.

[28] Christopher Edwards and Yuri B Shtessel. Adaptive Continuous Higher Order Sliding Mode Control. Automatica, 2016, 65:183-190.

[29] Haibin Dou. Lyapunov approach for motion synchronization of a two-slider system. Transactions of the Institute of Measurement and Control. 2019, 41(14): 4063-3072.

[30] Elinirina 1. Robinson, Julien Marzat and Tarek Raissi. Interval observer design for unknown input estimation of linear time-invariant discrete-time systems. IFAC-PapaersOnLine, 50(1):4021-4026.

[31]Tarek Raissi, Gaetan Videau and Ali Zolghadri. Interval observer design for consistency checks of nonlinear continuous-time systems. Automatica, 2010, 46(3):518-527.

[32]Hamed Rabiee, Mohammad Ataei and Mohsen Ekramian. Continuous nonsingular terminal sliding mode control based on adaptive sliding mode disturbance observer for uncertain nonlinear systems. Automatica, 2019, 109:1-7.

[33]Christopher Edwards and Chee Pin Tan. Sensor fault tolerant control using sliding mode observers. Control Engineering Practice, 2006, 14:897-908.

[34]Jinpeng Yu, Peng Shi and Lin Zhao. Finite-time command filtered backstepping control for a class of nonlinear systems. Automatica, 2018, 92: 173-180.
[35]A. Levant. Higher-order sliding modes, differentiation and output-feedback control. International Journal of Control, 76(9-10): 924-941.

[36]BeiBei Ren, Shuzhi Sam Ge, Keng peng Tee et al. Adaptive neural control for output feedback nonlinear systems using a barrier Lyapunov function. IEEE Transaction Neural Networks, 2010, 21(8):1339-1345. 